\title{
Virtual reality as an analgesic for acute and chronic pain in adults: a systematic review and meta- analysis
}

\author{
Brian Mallari' \\ Emily K Spaeth ${ }^{2}$ \\ Henry Goh ${ }^{3}$ \\ Benjamin S Boyd ${ }^{4}$ \\ 'Department of Physical Therapy, St. \\ Mary's Medical Center, San Francisco, \\ CA, USA; ${ }^{2}$ Castro Valley Yoga, Castro \\ Valley, CA, USA; ${ }^{3}$ Department of Physical \\ Therapy, Kaiser Permanente, Oakland, \\ CA, USA; ${ }^{4}$ Department of Physical \\ Therapy, Samuel Merritt University, \\ Oakland, CA, USA
} This article was published in the following Dove Press journal:
Journal of Pain Research

Background: Previous studies have shown that virtual reality (VR) is effective in reducing acute and chronic pain both in adults and in children. Given the emergence of new VR technology, and the growing body of research surrounding VR and pain management, an updated systematic review is warranted.

Purpose: The purpose of this systematic review is to compare the effectiveness of VR in reducing acute and chronic pain in adults.

Data Sources: A search was conducted in three databases (PubMed, CINAHL, Trip) using standardized search terms.

Study Selection: Twenty experimental and quasi-experimental trials published between January 2007 and December 2018 were included based on prespecified inclusion and exclusion criteria. Pain intensity was the primary outcome.

Data Extraction: We extracted data and appraised the quality of articles using either the PEDro or Modified Downs and Black risk of bias tools.

Data Synthesis: The majority of studies supported the use of VR to reduce acute pain both during the procedure and immediately after. Numerous studies found VR reduced chronic pain during VR exposure but there is insufficient evidence to support lasting analgesia. There was considerable variability in patient population, pain condition and dosage of VR exposure.

Limitations: Due to heterogeneity, we were unable to perform meta-analyses for all study populations and pain conditions.

Conclusions: VR is an effective treatment for reducing acute pain. There is some research that suggests VR can reduce chronic pain during the intervention; however, more evidence is needed to conclude that VR is effective for lasting reductions in chronic pain.

Keywords: virtual reality, analgesia, acute, chronic, pain management, adult

\section{Plain language summary}

Virtual reality (VR) could potentially help reduce pain in adults undergoing medical procedures or suffering with chronic pain. We performed a systematic review of the available research to see if VR is helpful at reducing pain. We found twenty research studies of various quality published from January 2007 to December 2018. Ten studies examined VR for acute pain conditions, including pain related to burns or medical procedures. Ten studies examined chronic pain, including musculoskeletal, neuropathic, mixed musculoskeletal and neuropathic or unspecified chronic pain conditions. Most of the studies found that VR helped to decrease acute pain during and immediately after various medical procedures. Some studies found VR reduced chronic pain when people were using the VR device and immediately
Correspondence: Benjamin S Boyd Department of Physical Therapy, Samuel Merritt University, 450 30th Street, Suite 3728, Oakland, CA 94609 , USA

Tel +I $5108799200 \times 7376$

$\mathrm{Fax}+15104572112$

Email bboydI@samuelmerritt.edu 
after but the decrease in pain did not last with the protocols tested to date. However, more research is needed to determine if VR can be effective for lasting reductions in people with chronic pain.

\section{Introduction}

Pain management is a growing health-care concern in the US. An estimated 100 million adults in the US suffer from chronic pain, and approximately $\$ 17.8$ billion is spent prescribing pain medication annually. ${ }^{1}$ Historically, opioid medications have been used for pain management, but there are negative side effects associated with opioid use, including a potential delay in recovery and increased risk of permanent disability. ${ }^{2}$ In addition, opioid use has been associated with increased hospital admissions, increased health-care costs, and deaths. ${ }^{2}$ Even with these risks and health-care burdens, the US consumes $80 \%$ of the world's opioids. $^{2}$ Alternative pain management strategies are important to consider in an effort to mitigate some of these risks.

A growing body of evidence supports VR as an alternative strategy for management of acute, burn, and experimental pain in adults and children. ${ }^{3-11}$ Additional experiments demonstrated the positive effect of virtual reality (VR) on pain during a variety of medical procedures including chemotherapy ${ }^{12-14}$ and wound care. ${ }^{15,16}$ Other studies also support VR as an adjunct to pain medications. ${ }^{17,18}$ Furthermore, evidence from systematic reviews ${ }^{17-21}$ conclude that VR is effective in the management of acute pain associated with medical procedures, wound debridement, and experimental pain.

Despite emerging evidence, consensus on the effectiveness of VR on chronic pain remains inconclusive. The most recent systematic review on VR both in acute and in chronic pain was performed over 8 years ago. ${ }^{19} \mathrm{~A}$ number of the studies included in that review are less applicable to the clinical setting because they explored the impact of VR on experimental pain, including painful thermal and pressure stimulation. Furthermore, much of the research has focused on children, ${ }^{5,711,16}$ which limits generalizability to adult populations. Finally, VR technology can be expensive and nonportable. However, the emergence of more affordable devices such as head mounted displays (HMD) has made VR more feasible for clinical use. Given these concerns, an updated systematic review comparing acute and chronic pain in adults is warranted.
The purpose of this systematic review is to compare the effectiveness of VR in reducing acute and chronic pain in adults compared to standard care without VR or to a sham treatment of nonVR.

\section{Materials and methods}

After preliminary searches, we performed our search using the combined terms "Virtual reality AND distraction", "Virtual reality AND pain", and "Virtual reality AND analgesia." It is hypothesized that distraction is a key mechanism for the effectiveness of VR, as the virtual environment consumes enough cognitive capacity to distract the user from their pain." ${ }^{3,22}$ Therefore, the term "distraction" was used to address the user's engagement in their virtual environment and potential mechanism of VR therapy, while the term "analgesia" was used to address pain reduction. Terms such as "immersive" were not included in our search strategy to allow a greater breadth of search as some studies did not make the distinction between immersive and nonimmersive VR. We performed searches using three databases: Turning Research into Practice (TRIP), CINAHL, and MEDLINE (via PubMed). No searches were performed to seek out gray literature. Full details of the search strategy and search filters used are provided in Appendix A.

For the purposes of this review, pain lasting shorter than three months was categorized as acute pain while ongoing, persistent pain lasting three months or longer was categorized as chronic pain. ${ }^{23,24}$ Researchers have defined VR as the interaction between a participant and a simulated threedimensional, immersive world. ${ }^{19}$ Previous research has compared immersive to nonimmersive VR and found immersive VR to be more effective in pain management. ${ }^{7,17-20,25-27}$ Thus, an inclusion criteria for this systematic review was "immersive" VR, defined by the participant being engaged in a simulated world in the form of visual and auditory feedback with the ability to interact with and/or elicit a reaction from their virtual environment. "Nonimmersive" VR and other distraction-based interventions, such as visual illusions, were exclusion criteria for this systematic review. This systematic review was registered in PROSPERO (CRD42018117881).

We screened articles in three stages. First, we screened titles and abstracts of each article and included them for full-text analysis based on the following criteria: written in English, published from January 2007 to December 2018, studied adults (18 years or older), used immersive VR, and used pain intensity as a primary outcome measure. Studies 
with a control group were included if the comparison treatment included standard care without VR or a sham treatment of nonVR. Nonexperimental studies were excluded, in addition to studies that used experimental pain, did not incorporate VR, or used VR for purposes outside of pain control. We excluded studies published before 2007 to attain the most current evidence using modern VR technologies. ${ }^{19}$

Second, two researchers evaluated the full text of each article to further determine inclusion or exclusion. The references of previously published systematic reviews on VR were also screened for articles that may have been missed in our search strategies. We excluded articles that did not meet the inclusion criteria. Third, if the decision to include or exclude was not clear, we flagged the article for further evaluation. We discussed each flagged article and eligibility was determined based on the overall efforts of the experimenters to (a) create an environment with visual, auditory, and tactile feedback, and (b) provide methods of interaction between the user and virtual environment. Any flagged articles that did not meet these considerations were excluded.

We extracted the following data from included articles: study design, population size and description, acute or chronic pain, outcome measure for pain (numeric rating scale, NRS; visual analog scale, VAS; graphic rating scale, and brief pain inventory), type of pain (musculoskeletal, neuropathic, burn, or procedural), and parameters of VR intervention including definition, description, condition duration, and comparison intervention (as applicable). We also extracted relevant quantitative data required to determine effect size (ES) for studies that had a comparison group. No ES was calculated for single arm studies. We evaluated the homogeneity of included studies and determined the appropriateness of subgrouping and pooling studies within this systematic review.

The primary outcome of interest was pain intensity, subgrouped into pain reported during the intervention or after the intervention for acute compared to chronic pain. For this analysis, data from between-group design studies (randomized controlled trials including cross over designs, and quasi-experimental studies with a comparison group) was utilized to compare the efficacy of VR to reduce pain compared to standard care or a sham VR treatment. Subgroupings were characterized as follows: acute pain during intervention, acute pain after intervention, chronic pain during intervention, and chronic pain after intervention. As a secondary analysis, studies presenting data of within-group pain intensity reductions were compared to determine expected magnitude of change from VR intervention in patients with acute and chronic pain.

For the meta-analysis, we included studies that used quantitative outcome measures for pain such as the NRS or VAS and that contained enough data to calculate an ES. We then calculated the percentage of total variation across studies that was due to heterogeneity rather than chance $\left(\mathrm{I}^{2}\right){ }^{28}$ If the pooled data was relatively heterogeneous (50\% or more), a random effects model was used, and if it was relatively homogenous (less than 50\%) a fixed effects model was used. ${ }^{28}$

In masked pairs we reviewed each study for risk of bias using the PEDro tool for randomized controlled trials and Modified Downs and Black (MD\&B) quality index for nonrandomized studies. ${ }^{29,30}$ We discussed discrepancies, and a third reviewer helped to determine a final score. PEDro scores of 6-10 are considered high quality, 4-5 fair quality, and scores less than or equal to 3 are of poor quality. $^{31}$ Scores of less than 14 on the MD\&B are suggested to be of lower quality. ${ }^{32}$

\section{Results}

A total of 485 articles were identified through database searches (Figure 1). After titles and abstracts were screened, 49 articles were eligible for full text review. After reviewing, a total of 20 articles were included in this systematic review (Table 1). Fourteen of the articles were randomized controlled trials (nine of which were crossover designs), while the other six were quasi-experimental studies (no comparison group). Ten studies examined VR for acute pain conditions, including three in pain related to burns (BP) and seven in medical procedure related pain (MPRP). Ten studies examined chronic pain, including three in musculoskeletal pain conditions (MSKP), four in neuropathic pain conditions (NP), one in a mixture of musculoskeletal and neuropathic pain conditions (MSKP-NP) and two in an unspecified pain condition (UnP). Results of the quality assessments demonstrated that all studies included in this systematic review were of fair to high quality (Table 1).

The VR equipment varied between studies. Eighteen studies used goggles/glasses or another form of HMD that displayed a virtual environment. One study used two 3D projectors (chronic NP), while another used a 2D screen but with dynamic visual cues that responded to changes in treadmill speed (chronic MSKP). 


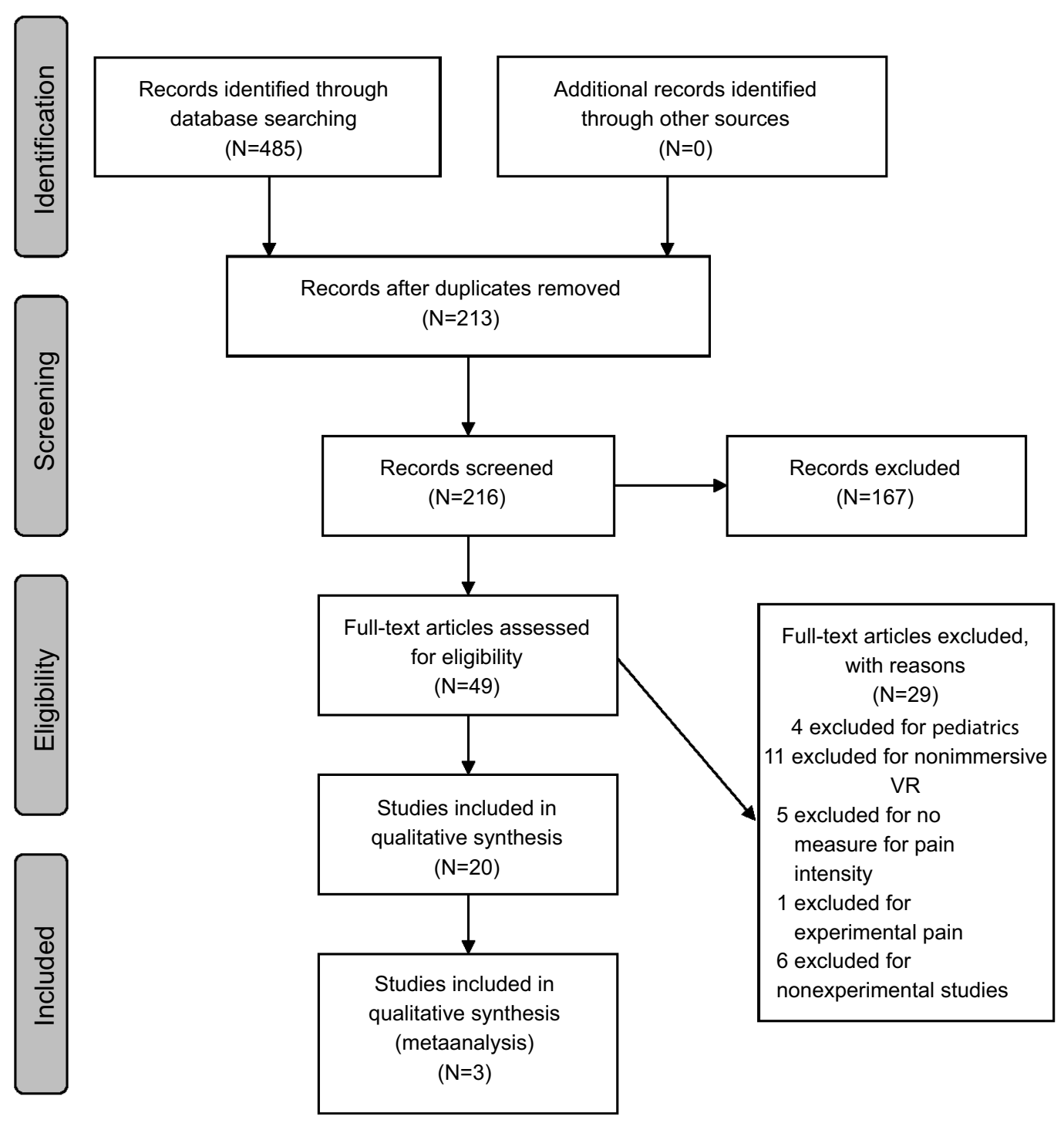

Figure I PRISMA flow diagram.

Abbreviation: VR, virtual reality.

\section{Acute pain conditions} Impact of VR on pain experienced during procedure Three studies examined BP and found that VR in conjunction with medications significantly reduced pain during wound debridement or remobilization of range of motion for limbs affected by burns compared to medications alone. ${ }^{9,25,33}$ Each of these studies used a HMD for VR application and had dosage between 6 and 20 minutes. Pooling of these studies was clinically appropriate due to the homogeneity of the study population and VR application. One study subdivided their sample into two groups based upon severity of worst pain (either $\geq 7 / 10$ or $<7 /$ 10). ${ }^{25}$ These independent groups were pooled together along with data from Carrougher, ${ }^{9}$ for the meta-analysis (Figure 2). However, there was insufficient data to calculate an ES from Morris et al, ${ }^{33}$ thus, this study was not included in the meta-analysis. The pooled ES was 0.66 (95\%CI: 0.20, 1.12) (Figure 2). Quality assessment scores for these studies were between 6-7 and 10 on the PEDro scale indicating high quality and low risk of bias.

Four studies evaluated the impact of VR on pain during various medical procedures (MPRP). ${ }^{34-37}$ One study found VR significantly reduced pain during periodontist procedures compared to both a control (standard care) $(\mathrm{ES}=0.95,95 \%$ CI: $0.64,1.26)$ and watching a movie during the procedure $(E S=0.44,95 \%$ CI: $0.23,0.65) .{ }^{34}$ Another study found VR significantly reduced pain during multiple stages of episiotomy repairs (hymen repair ES $=0.74,95 \%$ CI: $0.43,1.04$ and skin repair $\mathrm{ES}=1.00,95 \% \mathrm{CI}: 0.65,1.36$ ) in women after having their first baby compared to repair with no VR. ${ }^{35}$ 


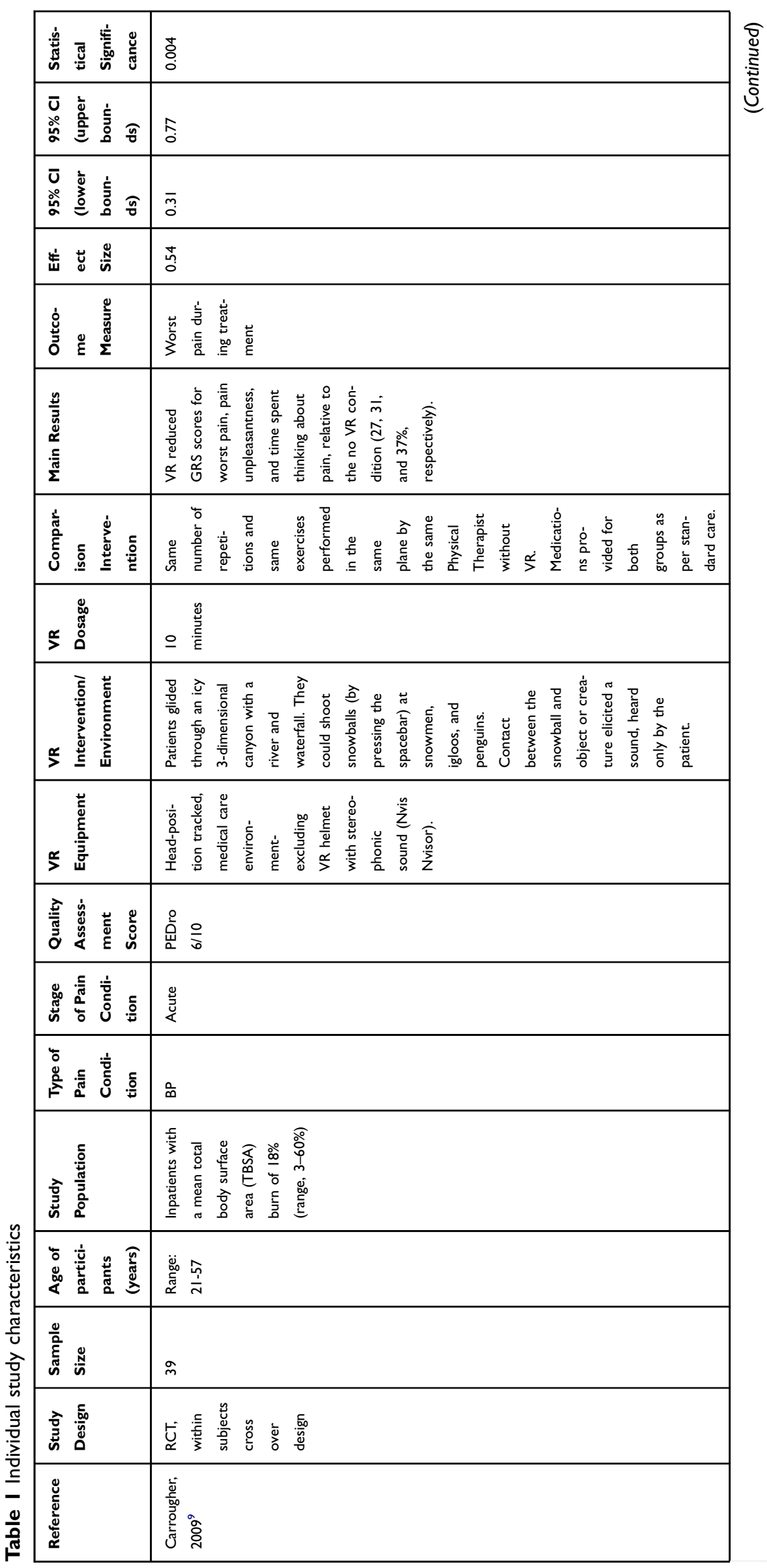




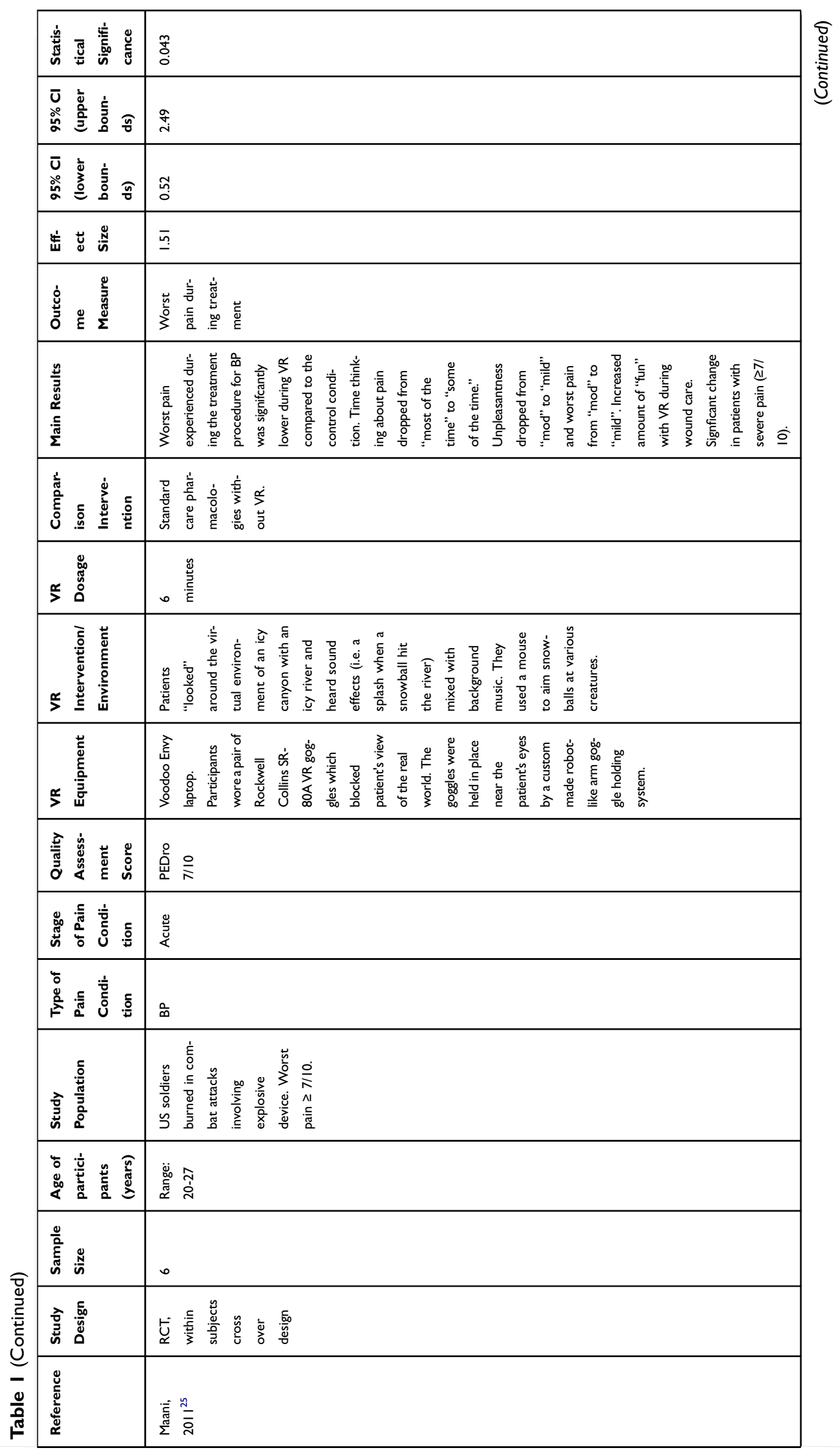




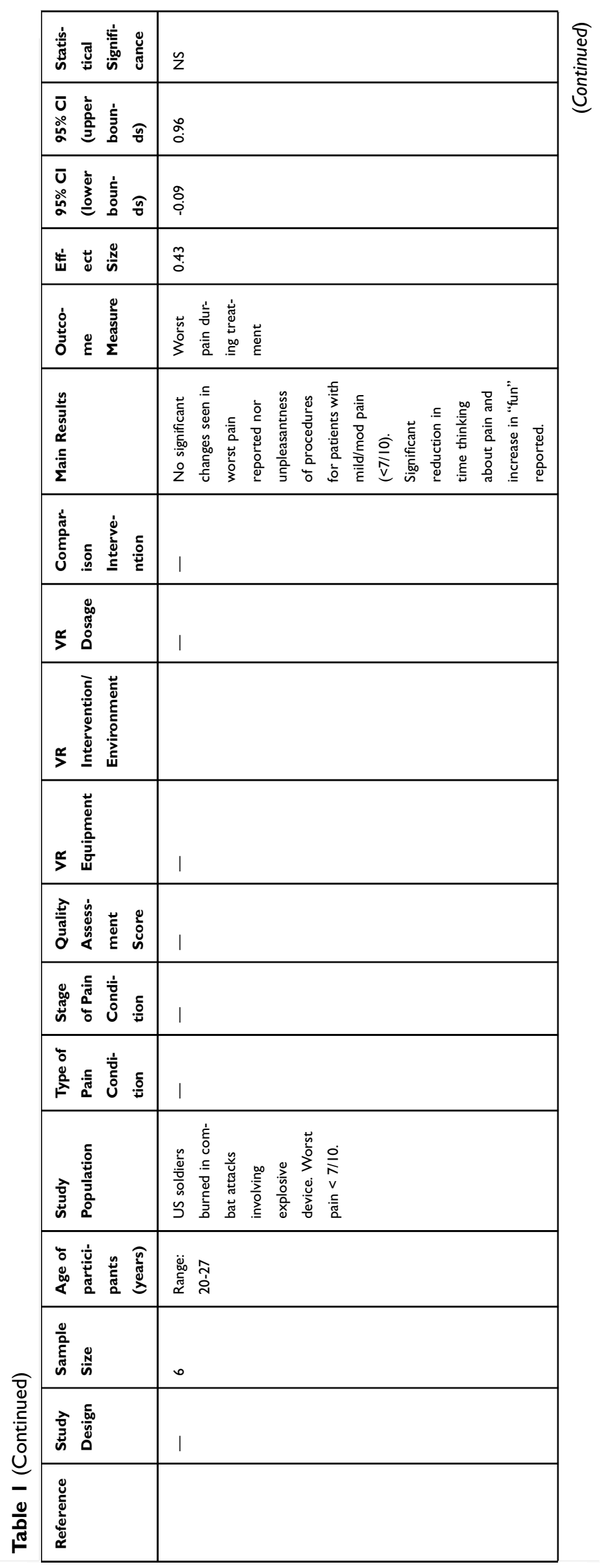




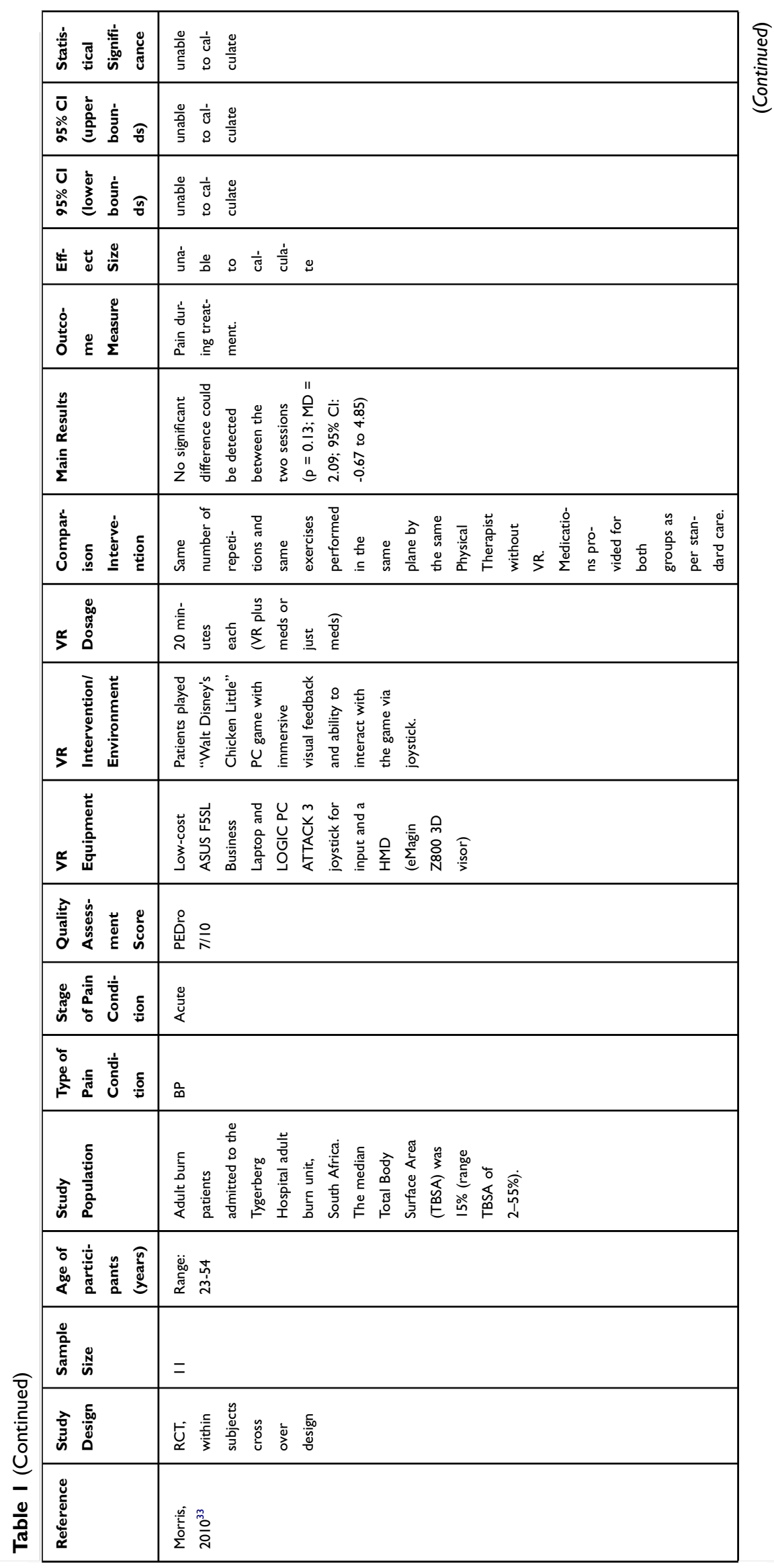




\begin{tabular}{|c|c|}
\hline 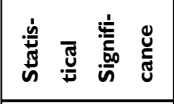 & 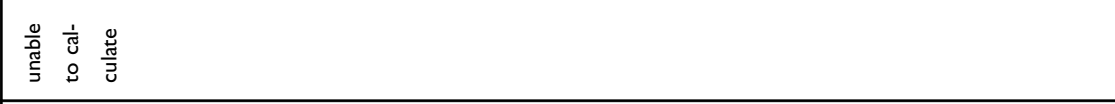 \\
\hline 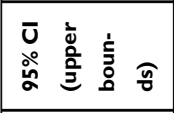 & 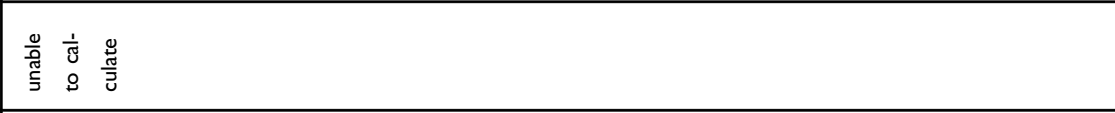 \\
\hline 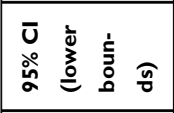 & 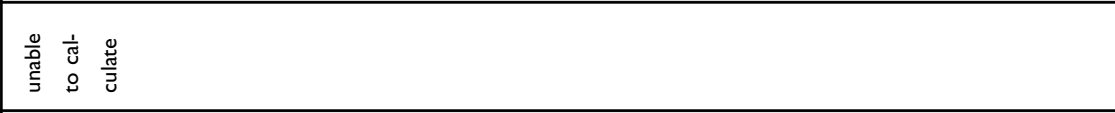 \\
\hline 岦 せ & 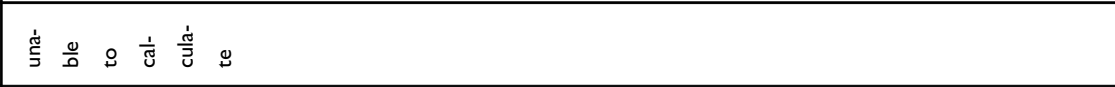 \\
\hline 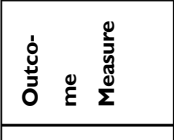 & 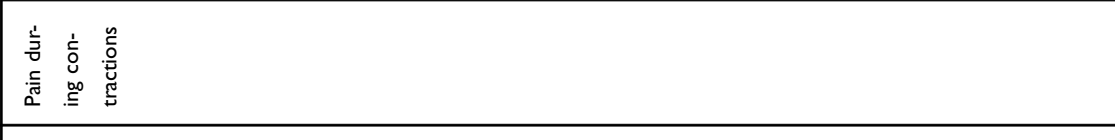 \\
\hline 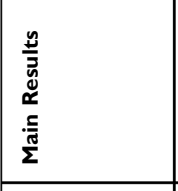 & 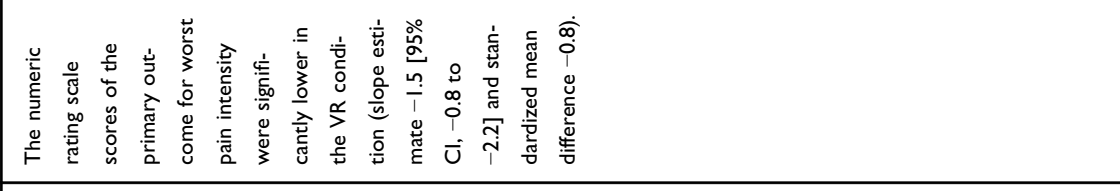 \\
\hline 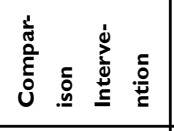 & 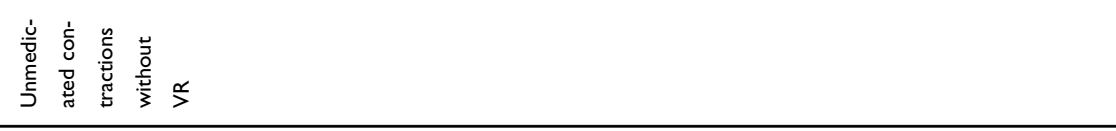 \\
\hline 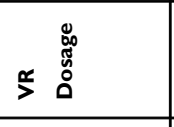 & 을 \\
\hline 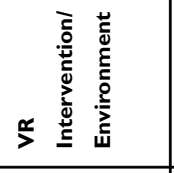 & 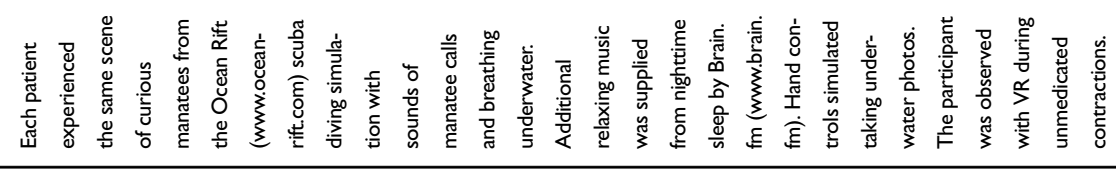 \\
\hline 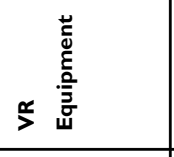 & 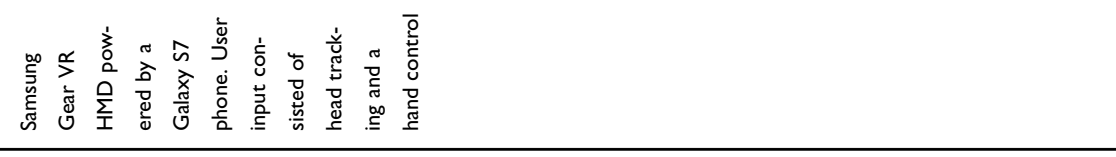 \\
\hline 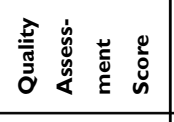 & 䢒 \\
\hline 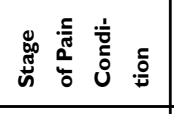 & $\frac{2}{3}$ \\
\hline 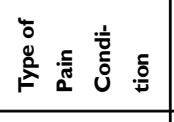 & $\frac{a}{\frac{\alpha}{\Sigma}}$ \\
\hline 旁高 & 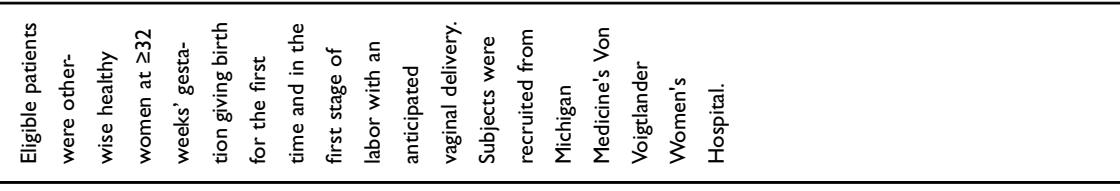 \\
\hline 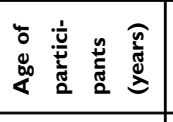 & 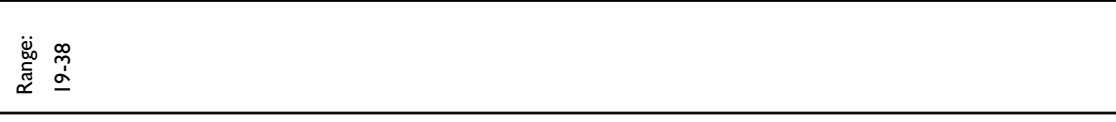 \\
\hline 迹 & ה \\
\hline 旁高 & 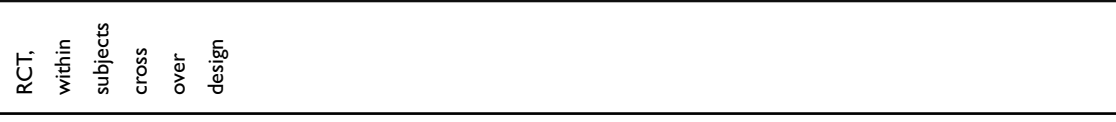 \\
\hline 這 & 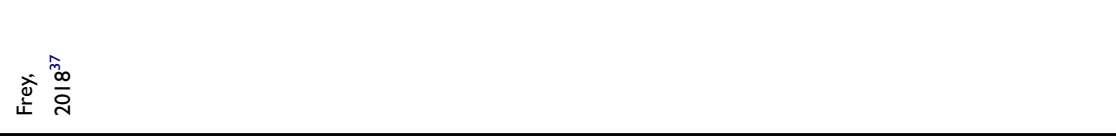 \\
\hline
\end{tabular}




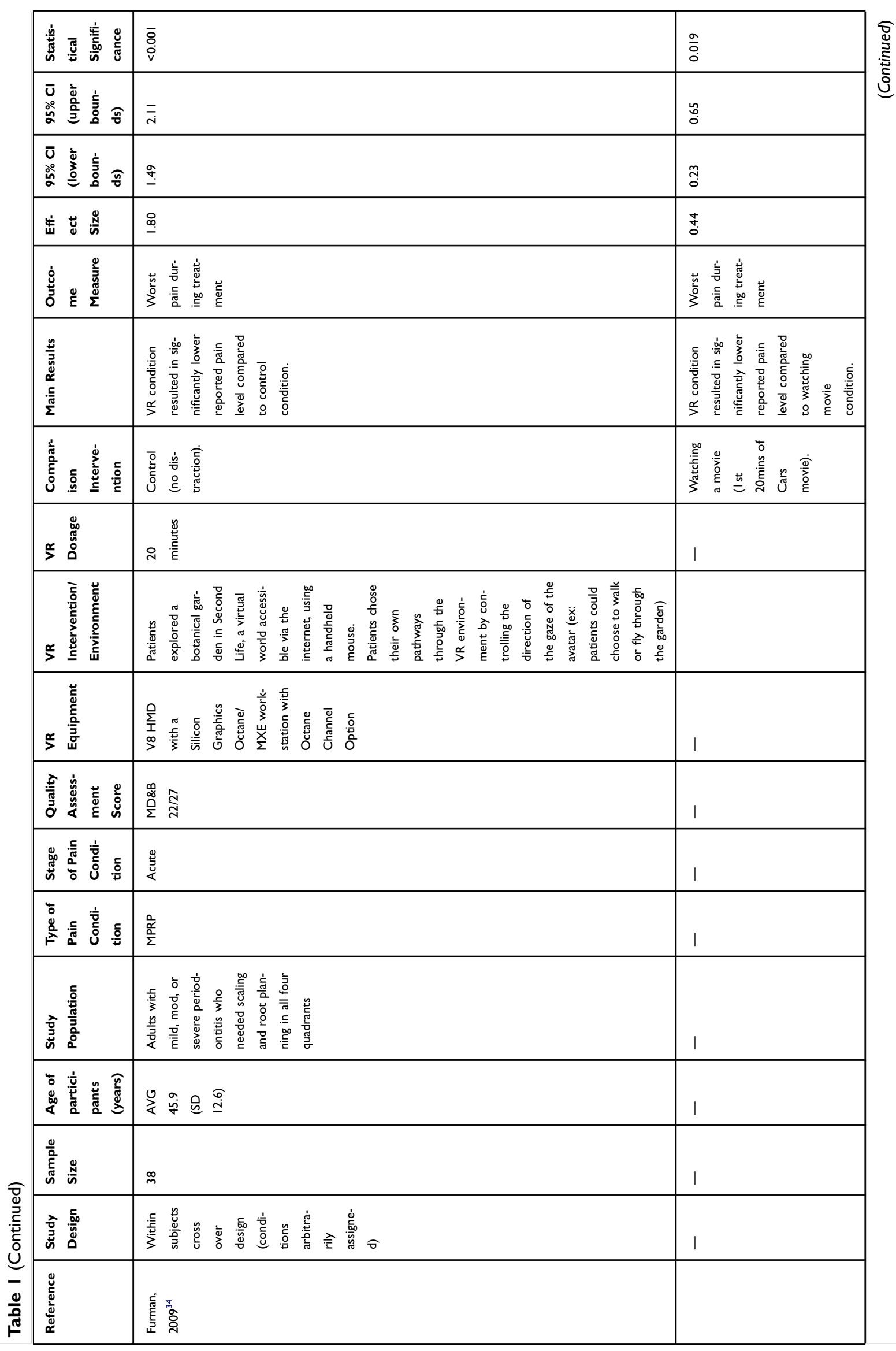




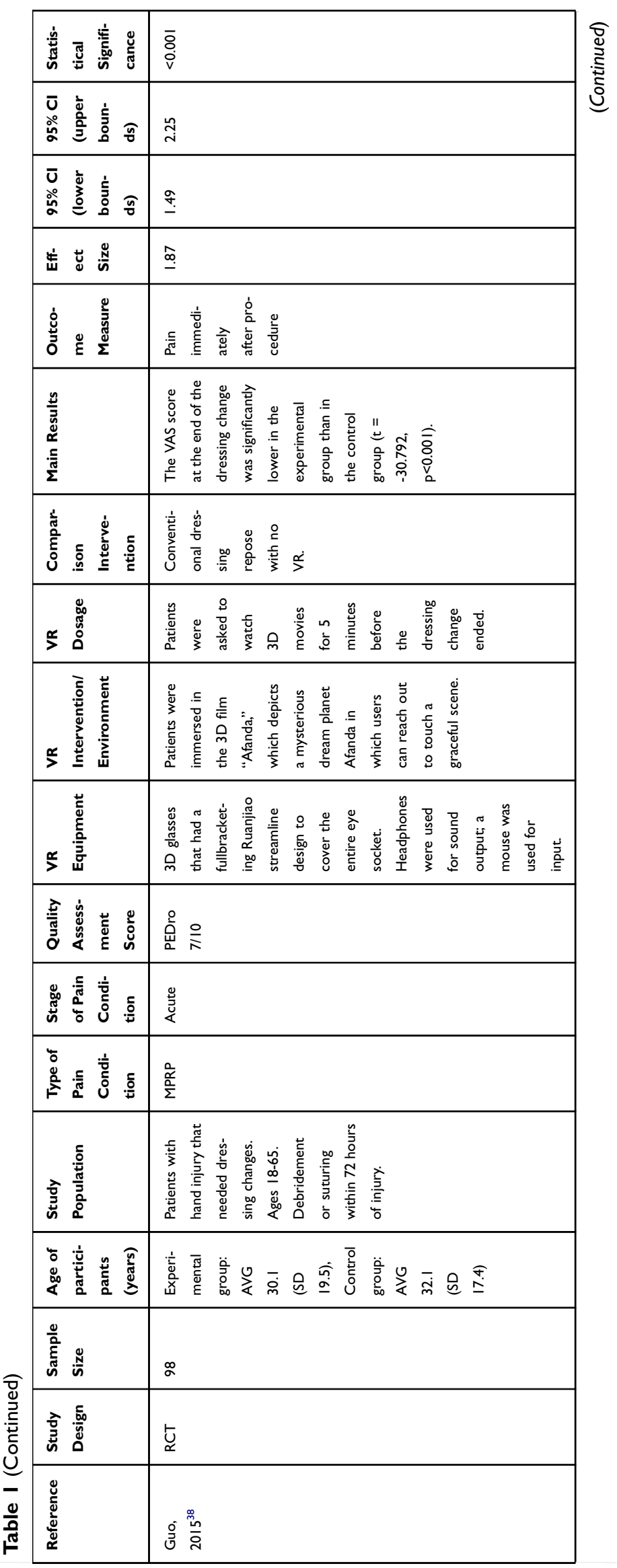




\begin{tabular}{|c|c|c|}
\hline 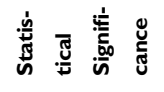 & $\stackrel{\infty}{0}$ & 怘 \\
\hline 立高 & $\stackrel{+}{\stackrel{+}{0}}$ & 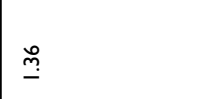 \\
\hline 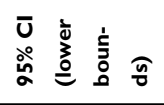 & m. & مْ \\
\hline 立 せัّ & $\underset{0}{t}$ & 8 \\
\hline 密 & 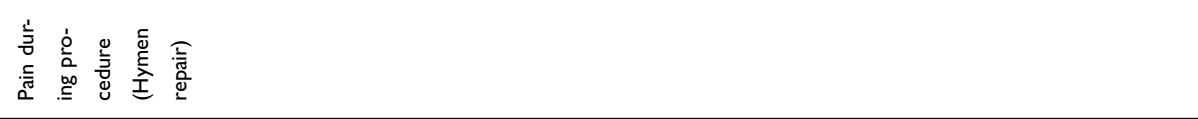 & 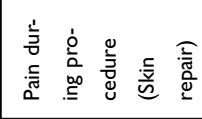 \\
\hline 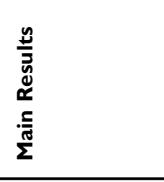 & 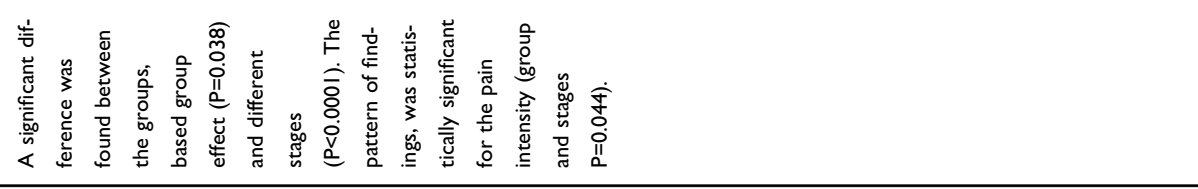 & I \\
\hline 总 & 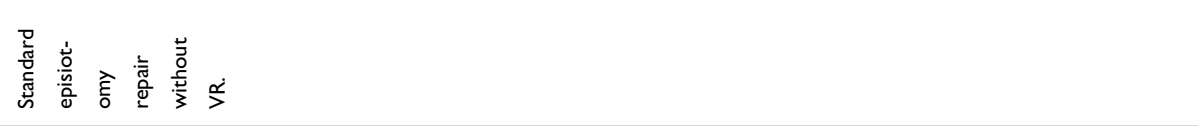 & 1 \\
\hline 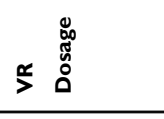 & 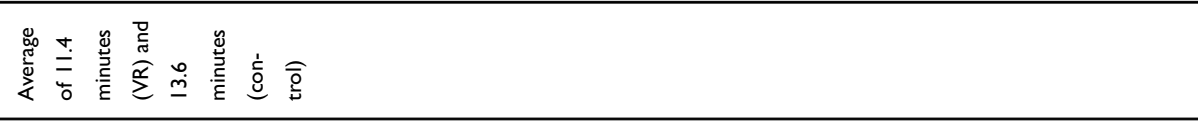 & 1 \\
\hline 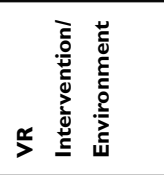 & 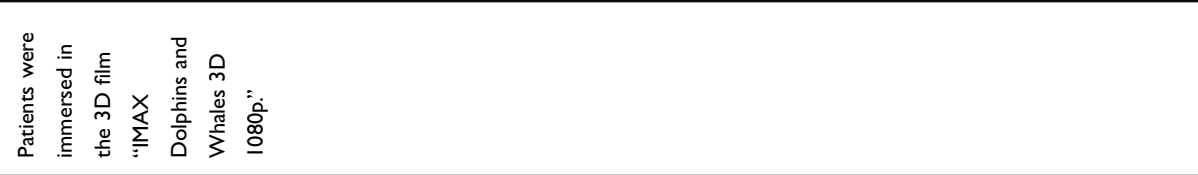 & \\
\hline 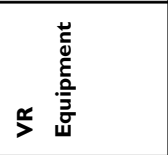 & 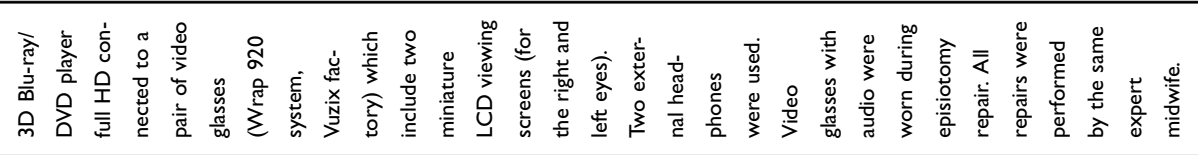 & I \\
\hline 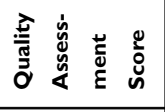 & 웄음 & 1 \\
\hline 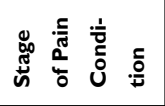 & $\stackrel{\Xi}{\check{z}}$ & I \\
\hline 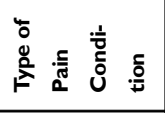 & $\frac{a}{\frac{\alpha}{\Sigma}}$ & I \\
\hline 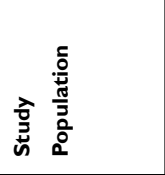 & 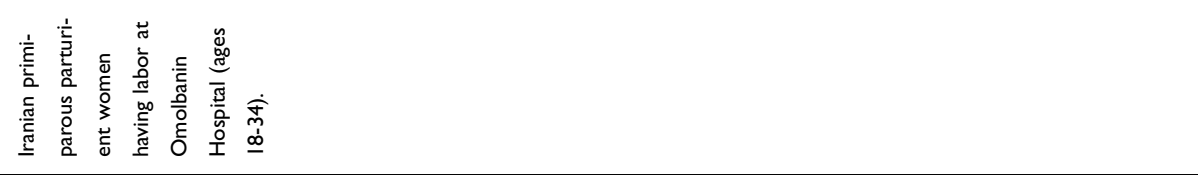 & 1 \\
\hline 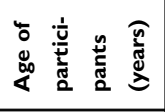 & 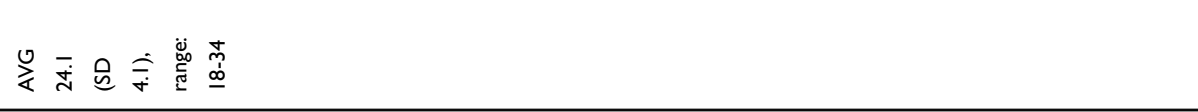 & I \\
\hline 造 & i & I \\
\hline 害高 & $\underset{\longleftarrow}{\longleftarrow}$ & I \\
\hline 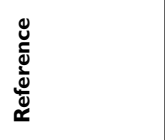 & 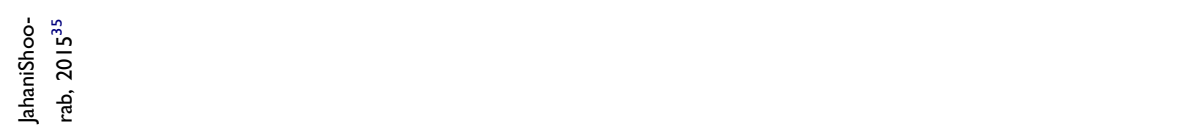 & \\
\hline
\end{tabular}




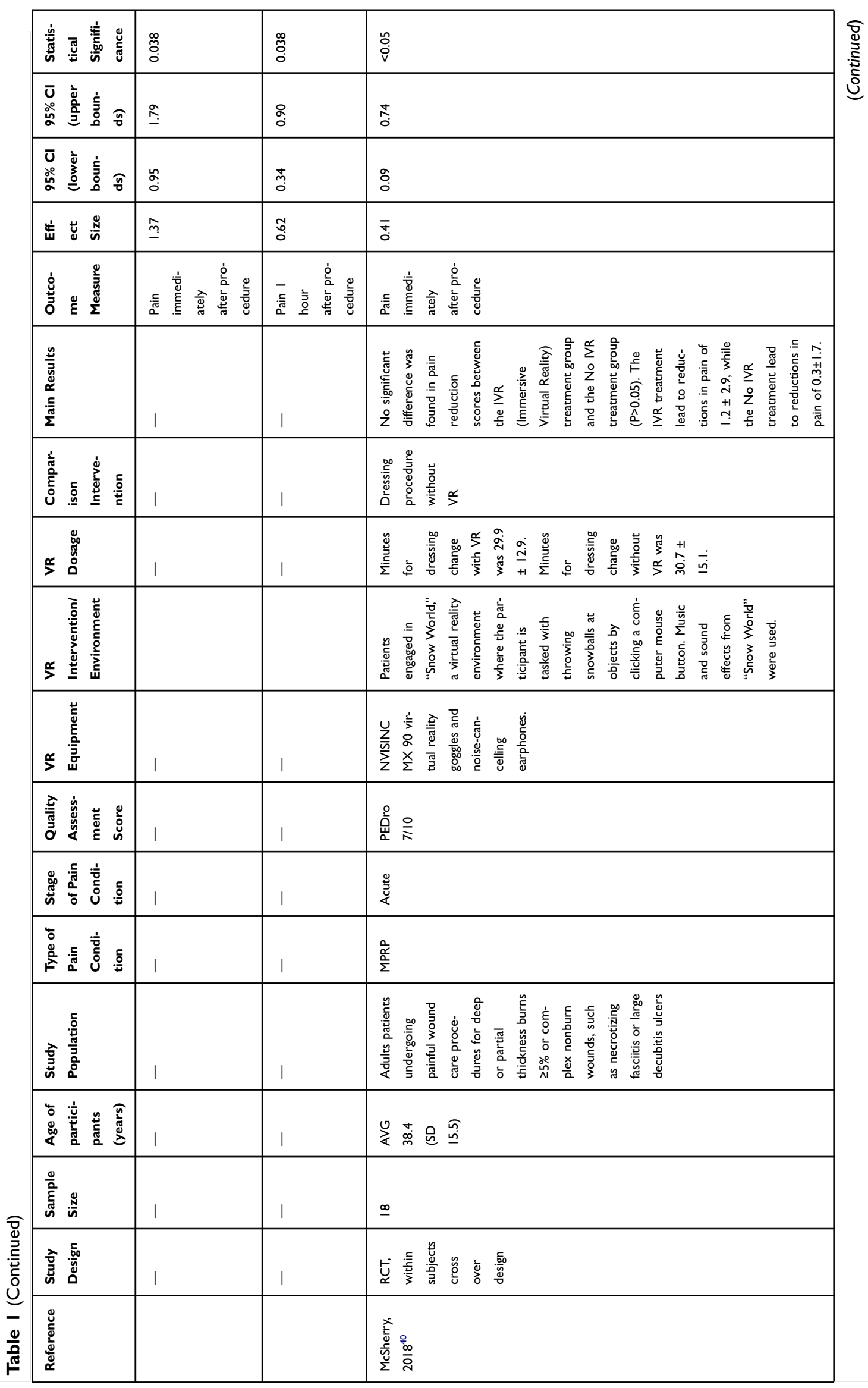




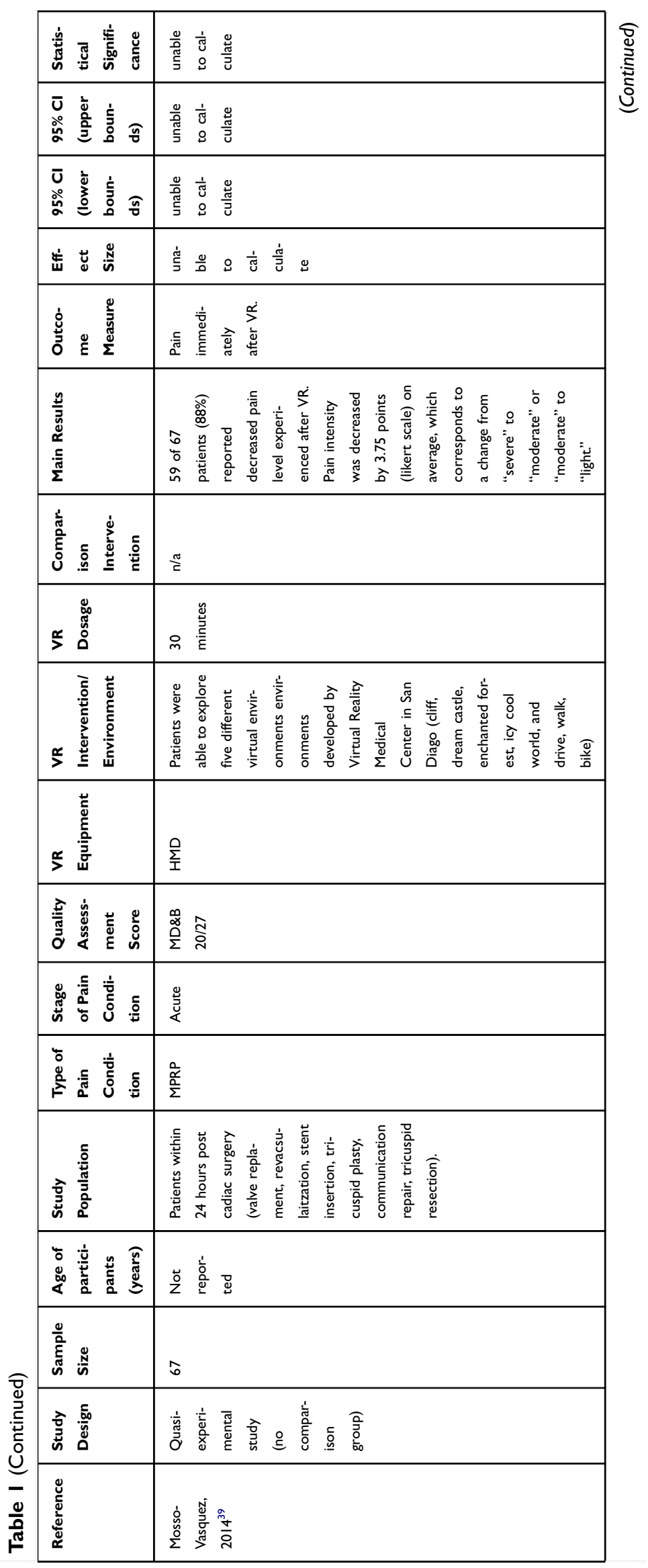




\begin{tabular}{|c|c|c|}
\hline 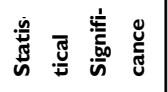 & 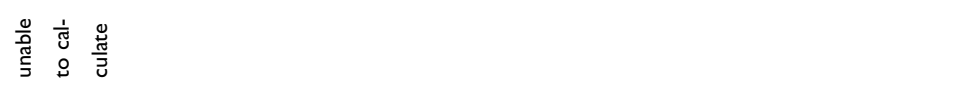 & 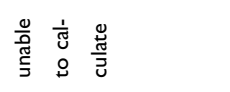 \\
\hline 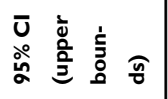 & 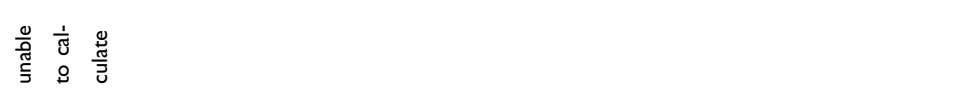 & 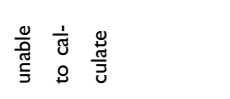 \\
\hline 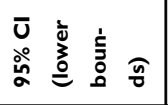 & 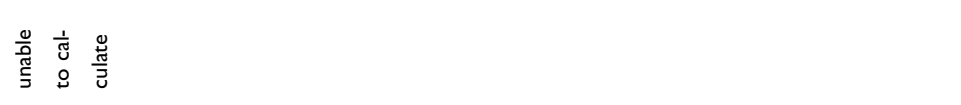 & 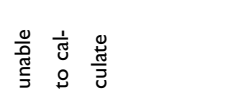 \\
\hline 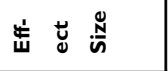 & 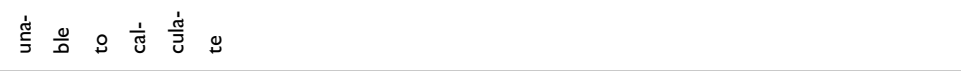 & 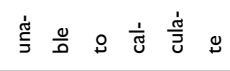 \\
\hline 乌ัٌ & 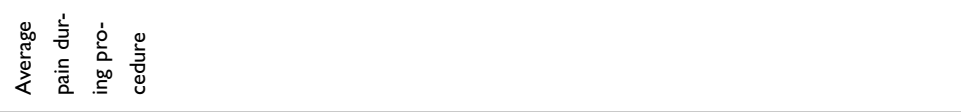 & 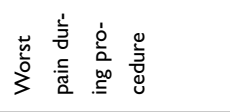 \\
\hline & 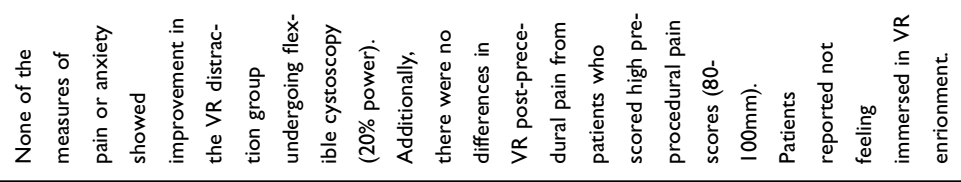 & 1 \\
\hline 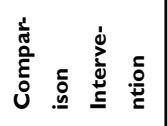 & 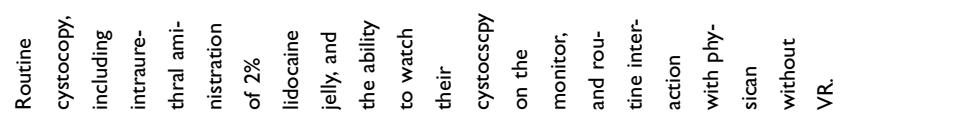 & | \\
\hline 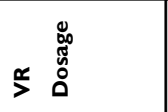 & 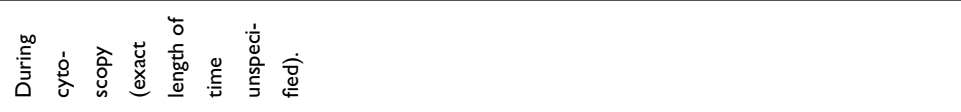 & 1 \\
\hline 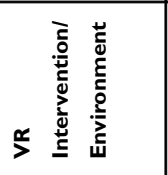 & 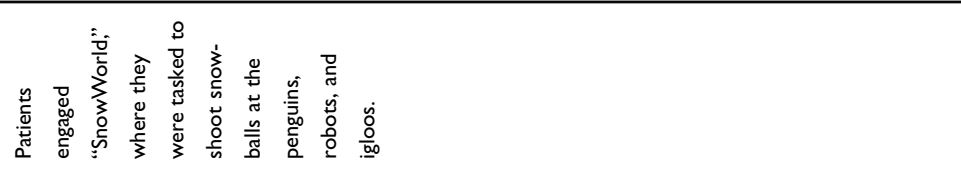 & \\
\hline 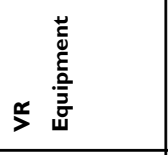 & 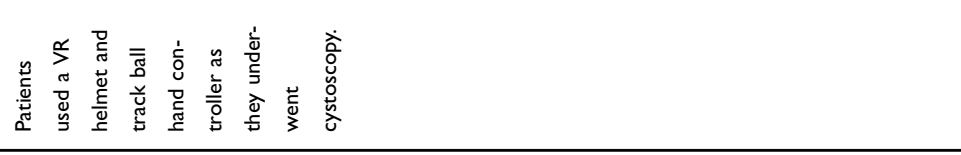 & । \\
\hline 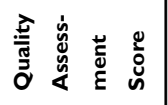 & 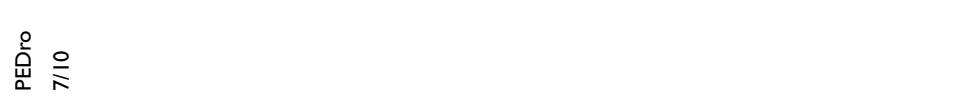 & 1 \\
\hline 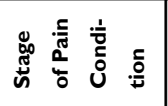 & $\stackrel{\Xi}{\ddot{\Sigma}}$ & I \\
\hline 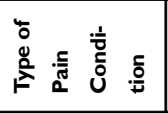 & $\frac{a}{\grave{\alpha}}$ & I \\
\hline 竞 & 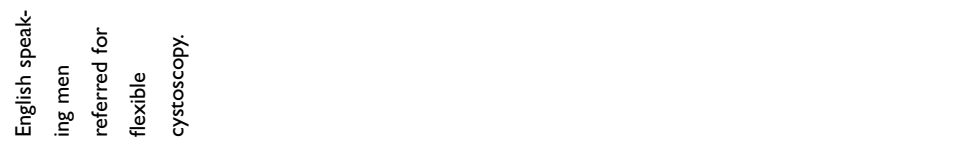 & । \\
\hline 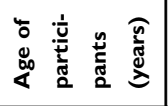 & 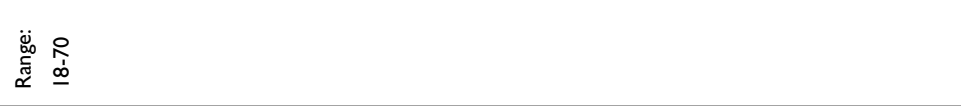 & । \\
\hline 造 & ซ & । \\
\hline 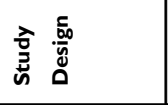 & $\underset{\check{c}}{5}$ & । \\
\hline 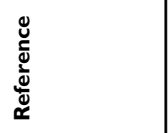 & 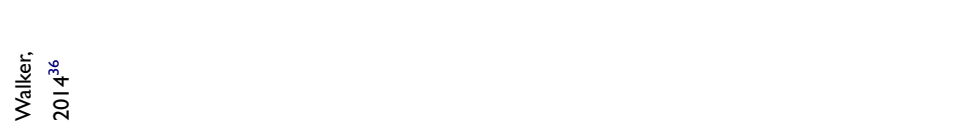 & \\
\hline
\end{tabular}




\begin{tabular}{|c|c|}
\hline 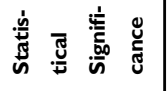 & 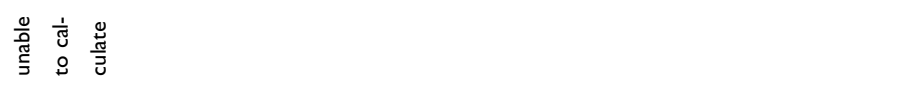 \\
\hline 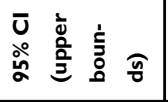 & 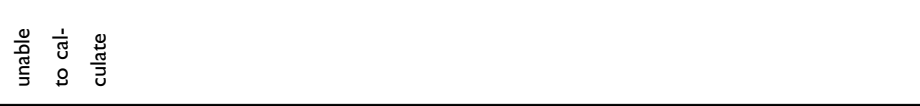 \\
\hline 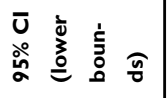 & 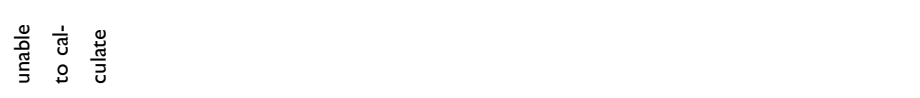 \\
\hline 姜 䓀 & 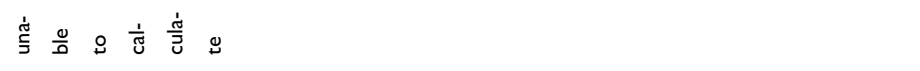 \\
\hline 㝘 & 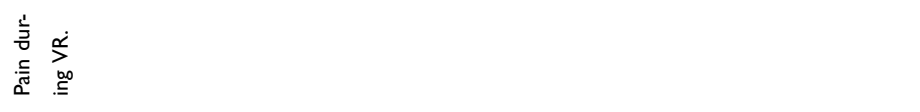 \\
\hline 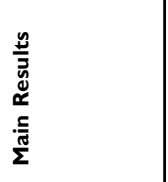 & 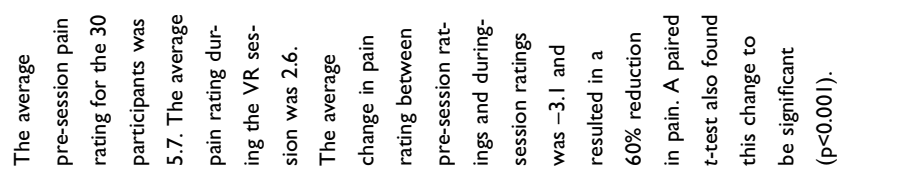 \\
\hline 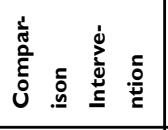 & 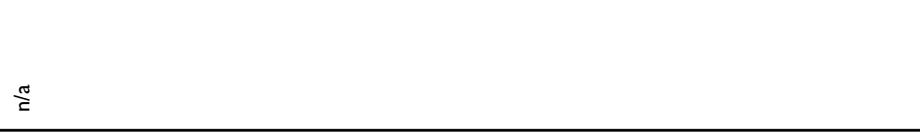 \\
\hline 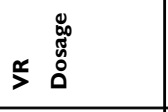 & n \\
\hline 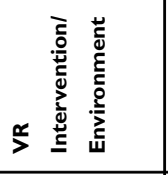 & 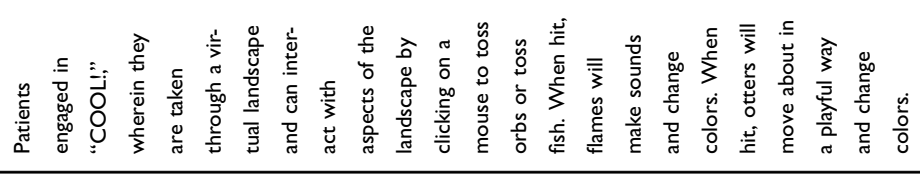 \\
\hline 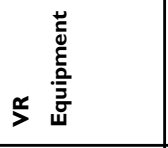 & 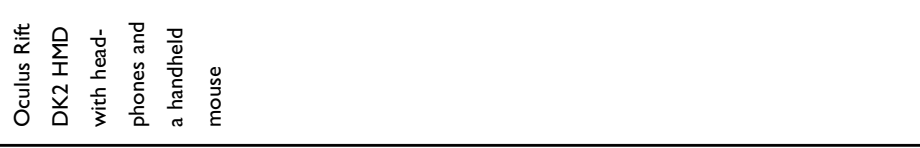 \\
\hline 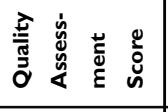 & 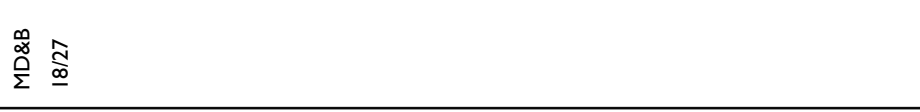 \\
\hline 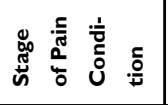 & 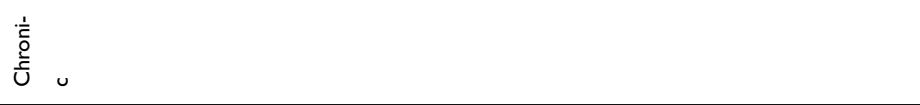 \\
\hline 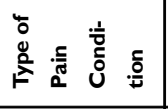 & $\frac{\dot{1}}{\frac{\hat{v}}{\Sigma}} \frac{\hat{z}}{\mathrm{z}}$ \\
\hline 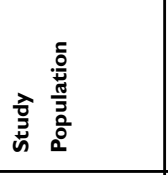 & 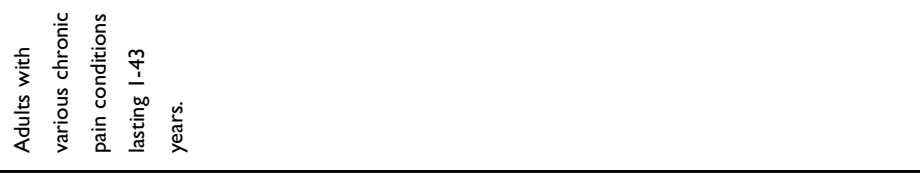 \\
\hline 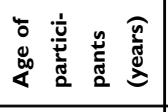 & 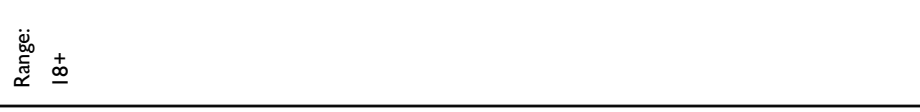 \\
\hline 资 & i \\
\hline 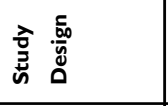 & 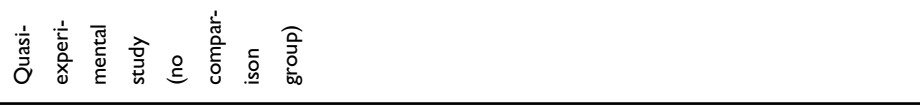 \\
\hline 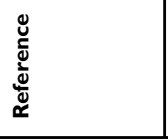 & 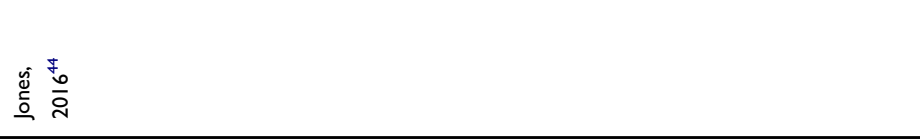 \\
\hline
\end{tabular}




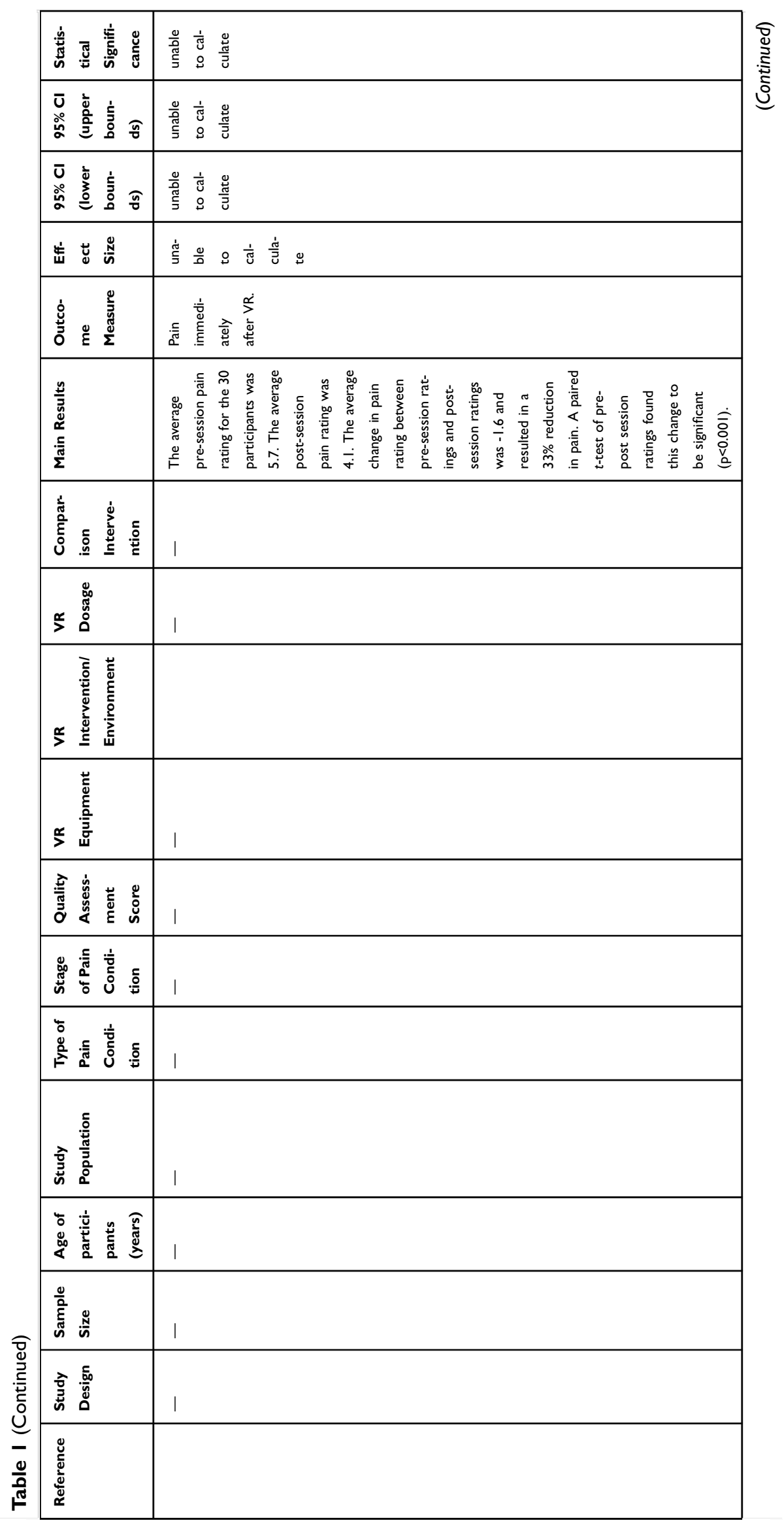




\begin{tabular}{|c|c|}
\hline 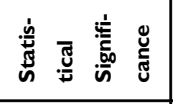 & 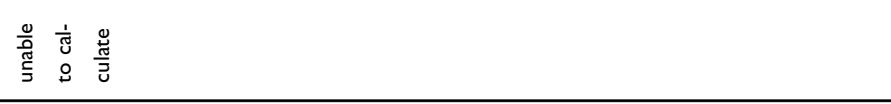 \\
\hline 可高高 & 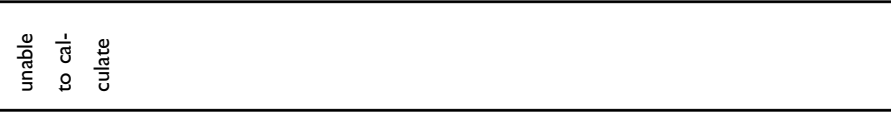 \\
\hline 可高高 & 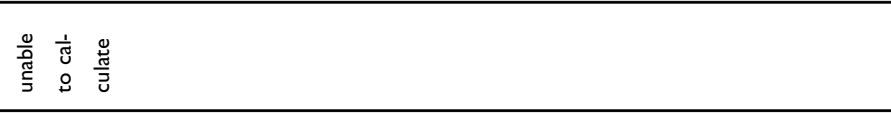 \\
\hline 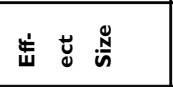 & 咅 。 \\
\hline 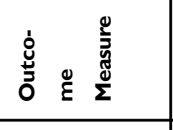 & 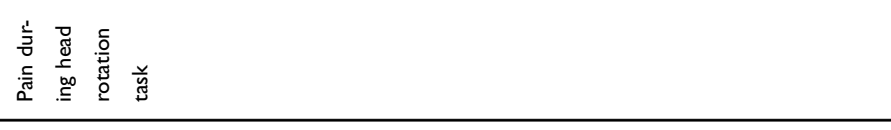 \\
\hline & 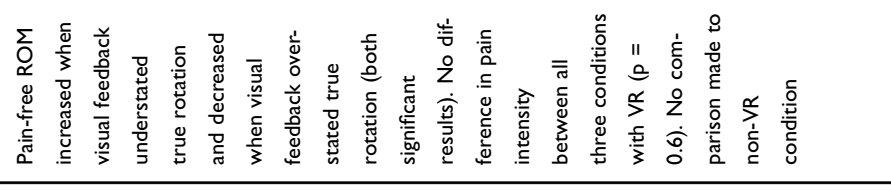 \\
\hline 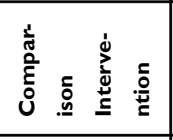 & 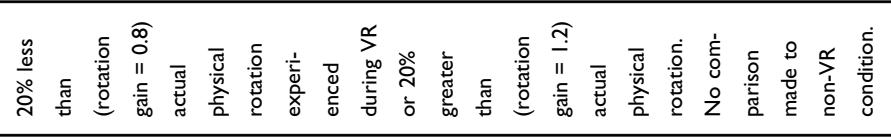 \\
\hline 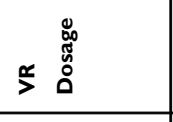 & 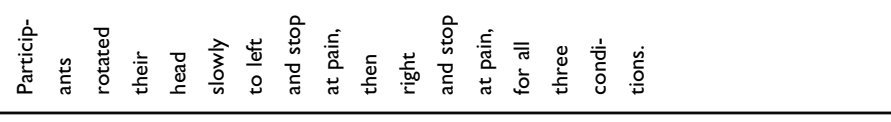 \\
\hline 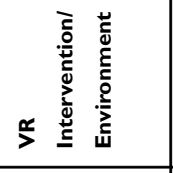 & 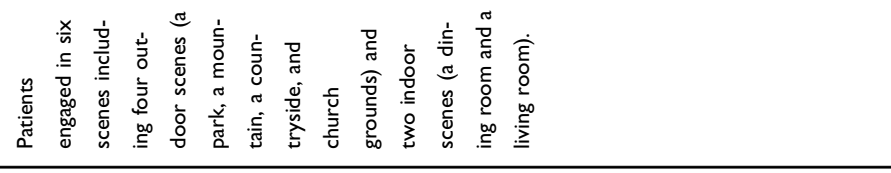 \\
\hline 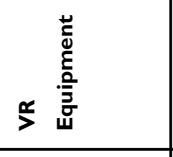 & 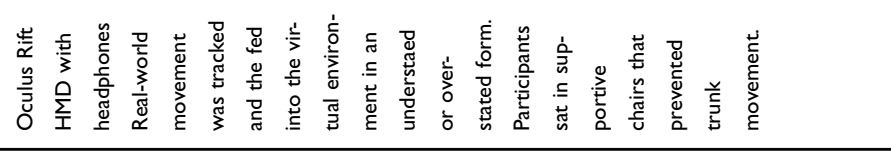 \\
\hline 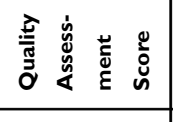 & $\stackrel{\circ}{\stackrel{8}{2} \frac{\circ}{\bar{\omega}}}$ \\
\hline 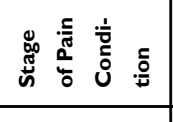 & 言 \\
\hline 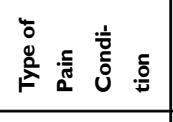 & $\frac{\partial}{2}$ \\
\hline 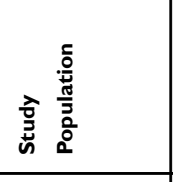 & 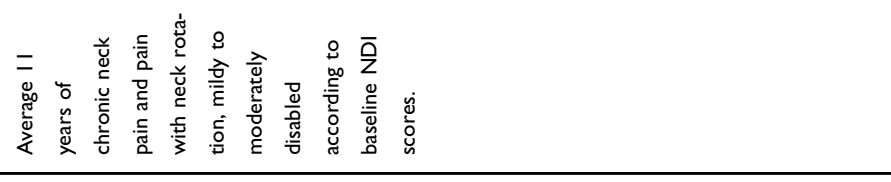 \\
\hline 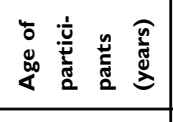 & 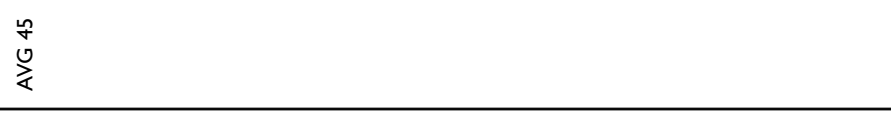 \\
\hline 迹 & \pm \\
\hline 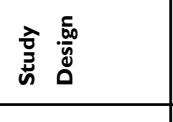 & 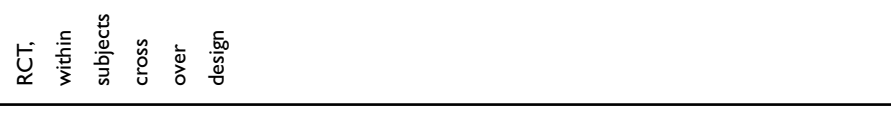 \\
\hline & 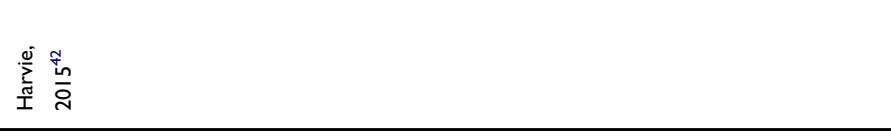 \\
\hline
\end{tabular}




\begin{tabular}{|c|c|}
\hline 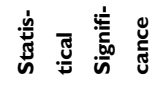 & 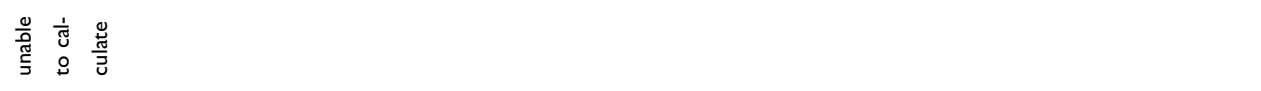 \\
\hline 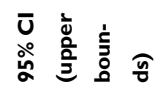 & 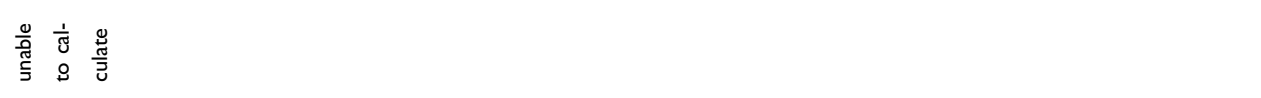 \\
\hline 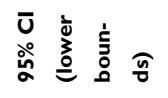 & 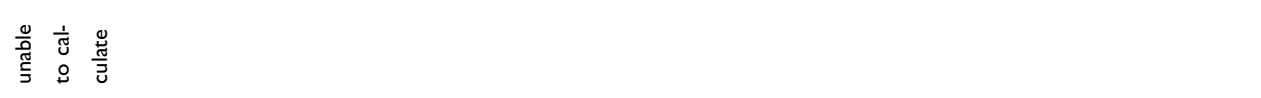 \\
\hline 岀 范 & 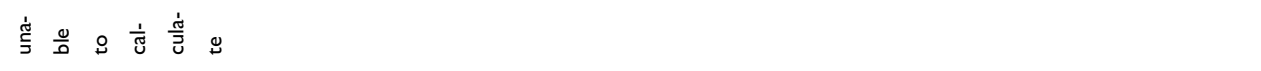 \\
\hline 乌ัٌ & 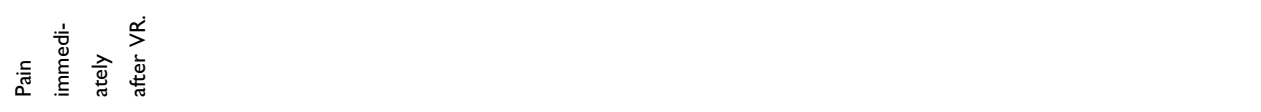 \\
\hline & 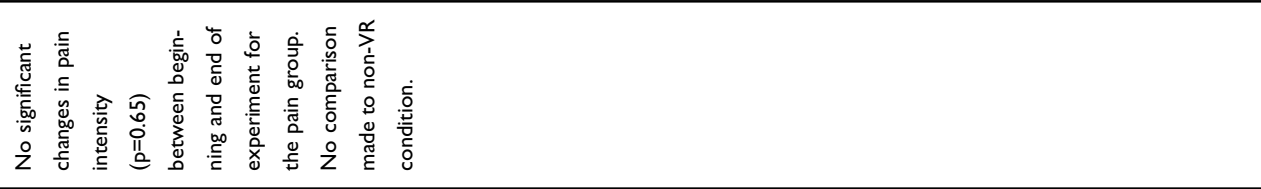 \\
\hline 总 & 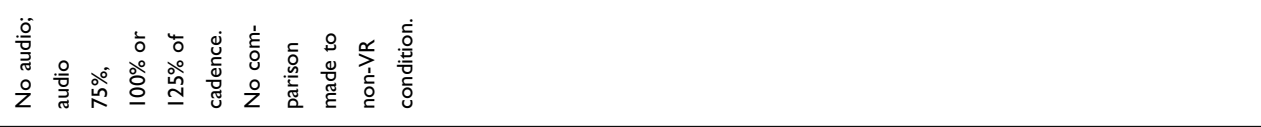 \\
\hline 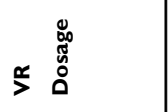 & 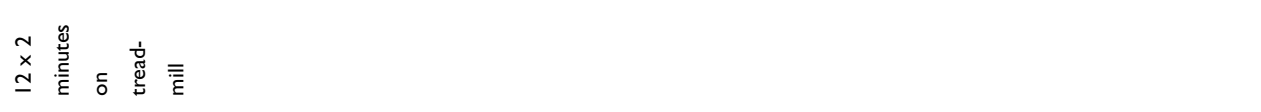 \\
\hline 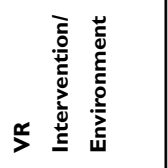 & 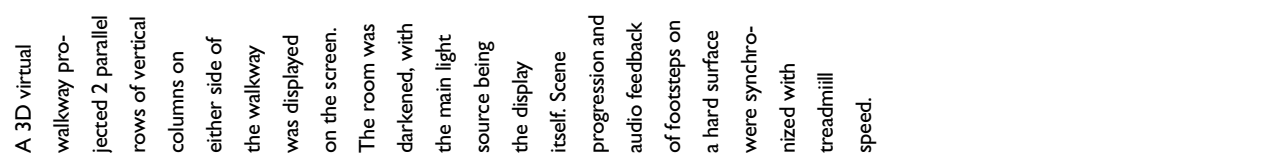 \\
\hline 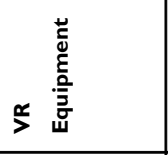 & 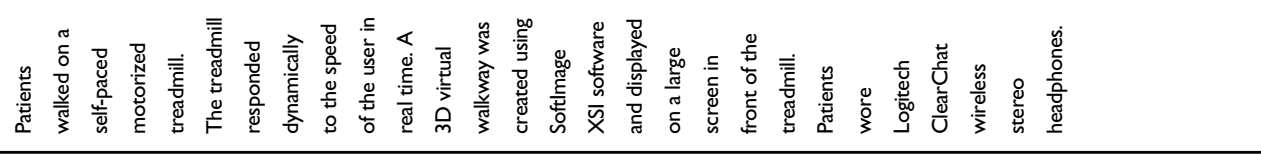 \\
\hline 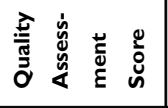 & 임음 \\
\hline 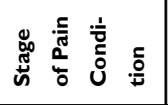 & 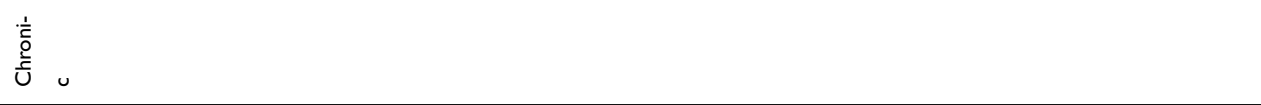 \\
\hline 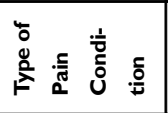 & $\frac{\hat{v}}{\Sigma}$ \\
\hline 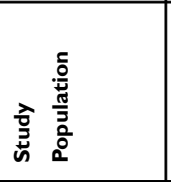 & 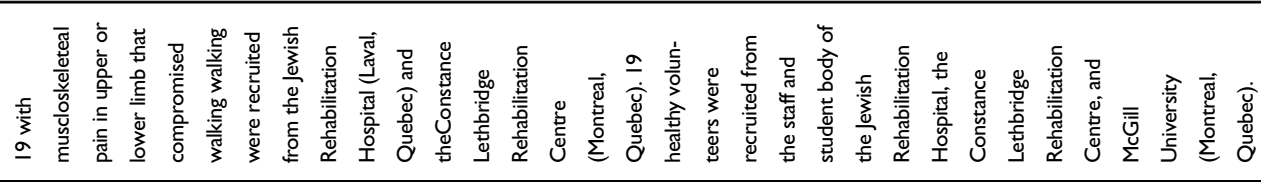 \\
\hline 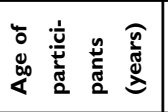 & 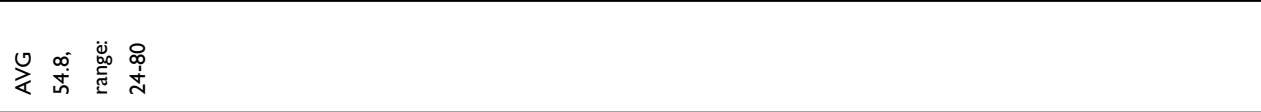 \\
\hline 造 & $\underline{a}$ \\
\hline 竧点 & 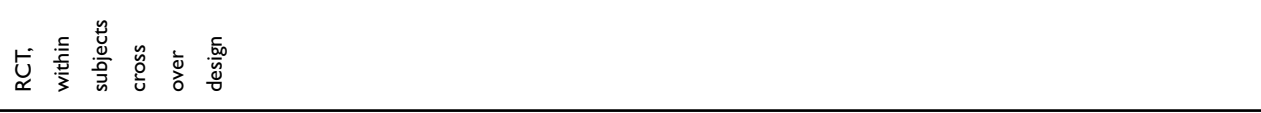 \\
\hline 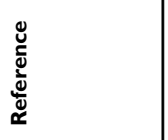 & 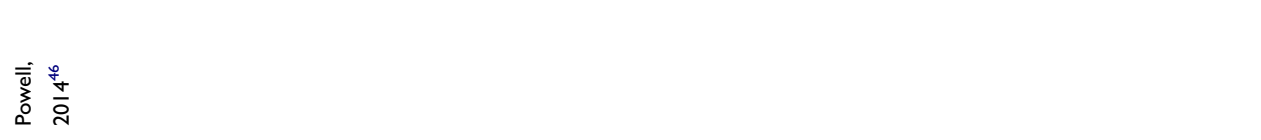 \\
\hline
\end{tabular}




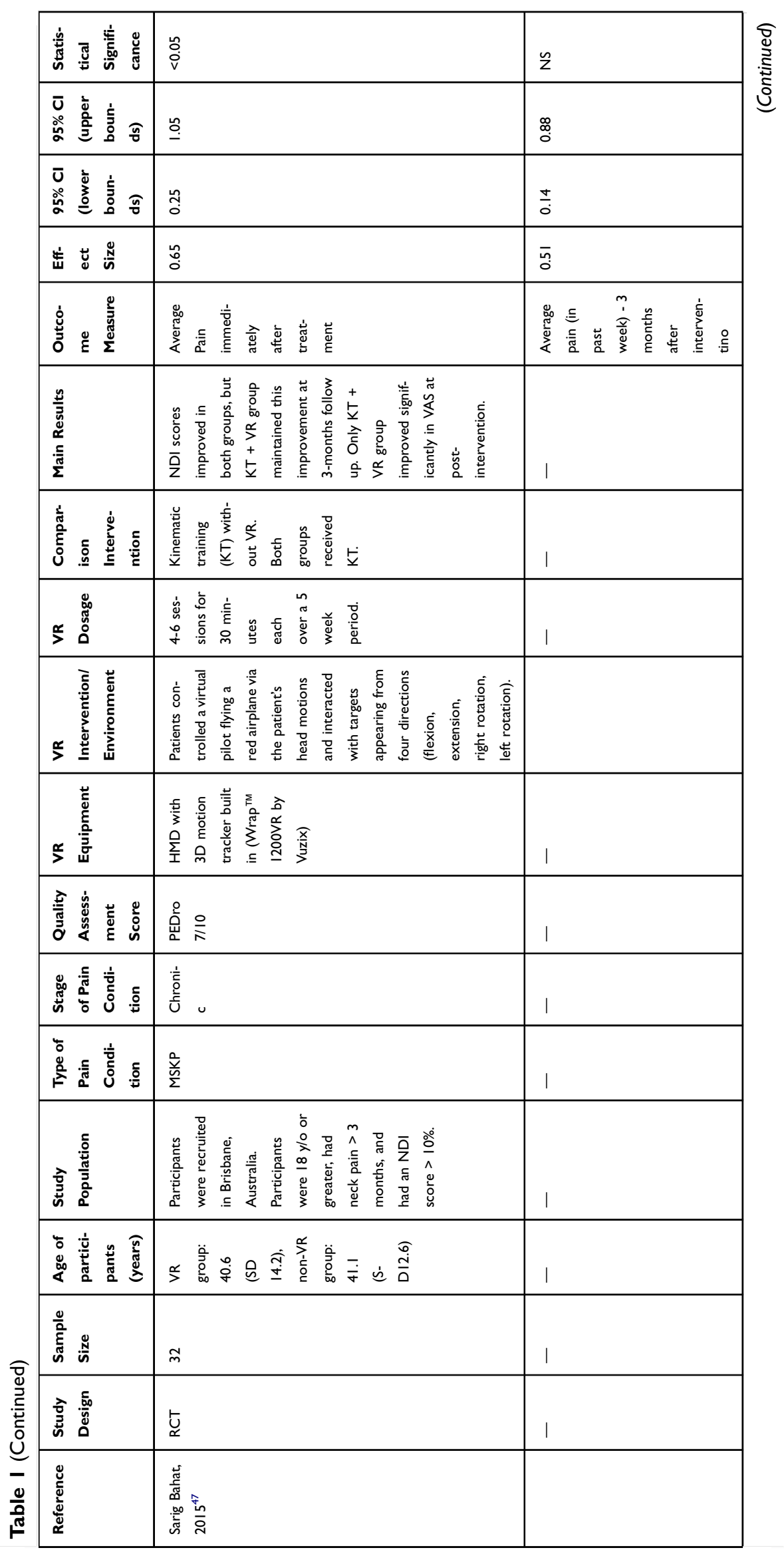




\begin{tabular}{|c|c|}
\hline 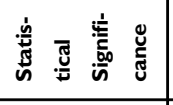 & 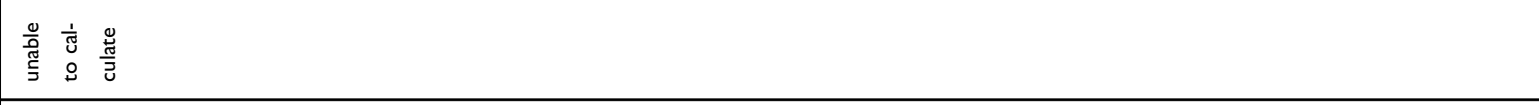 \\
\hline 可高高 & 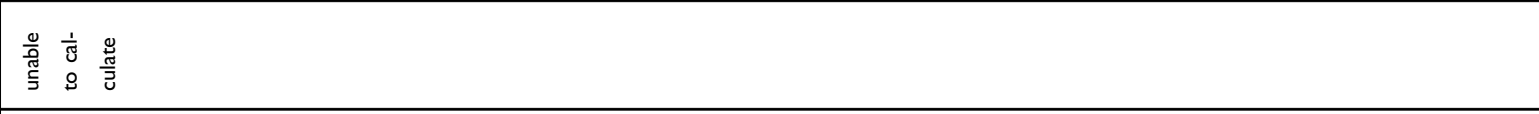 \\
\hline 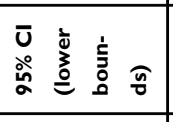 & 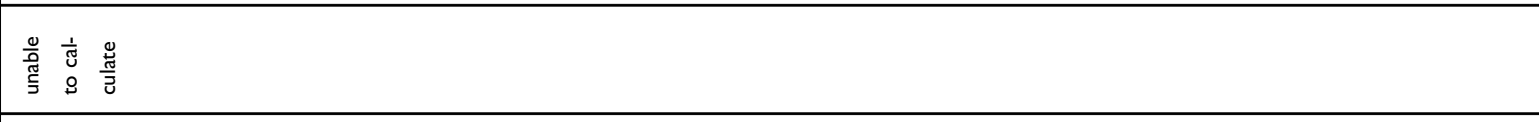 \\
\hline 岦 せัँ & 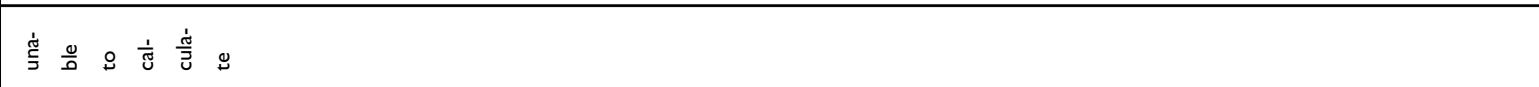 \\
\hline 乌ั & 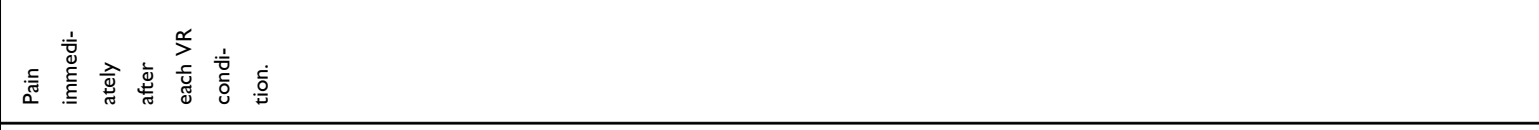 \\
\hline 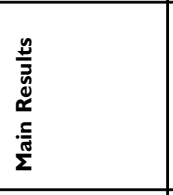 & 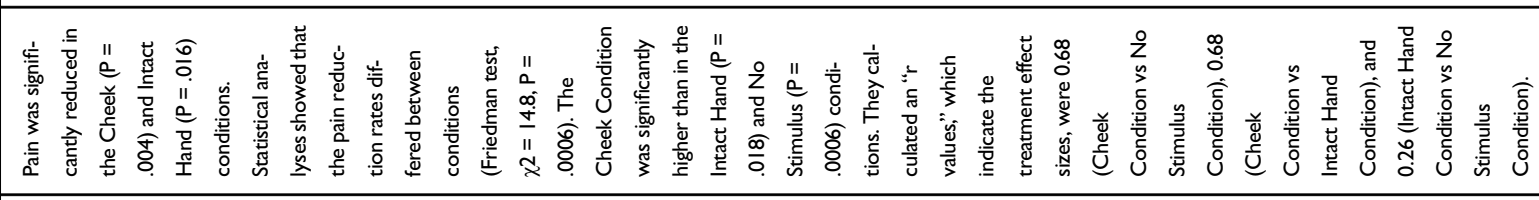 \\
\hline 总 & $\cong$ \\
\hline 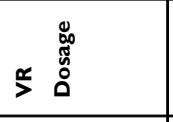 & 늘 \\
\hline 今 & 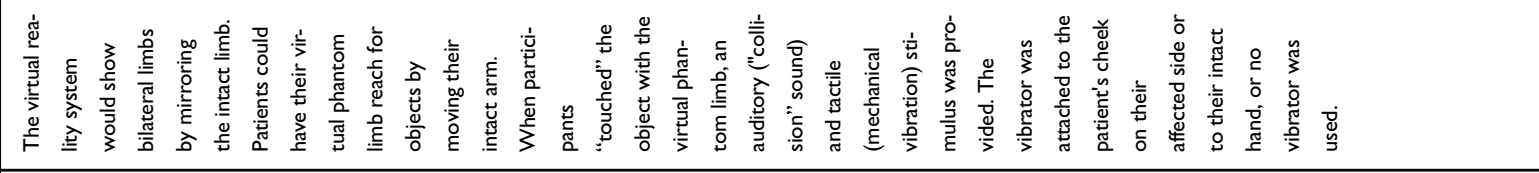 \\
\hline 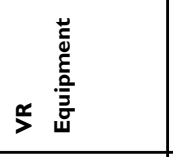 & 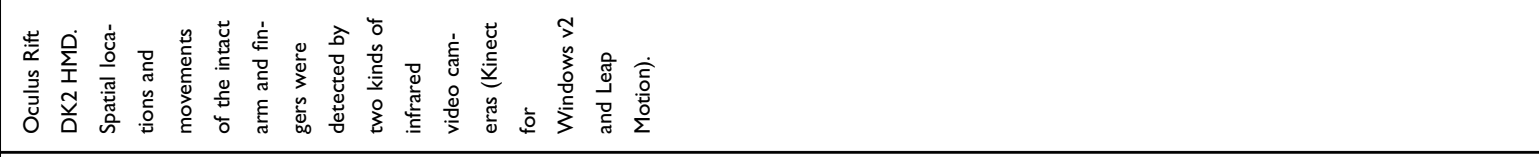 \\
\hline 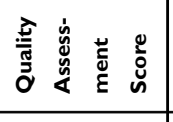 & 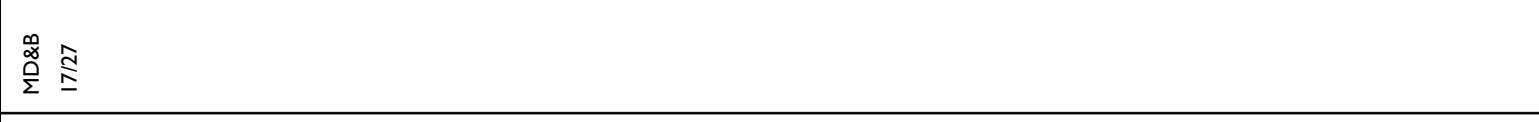 \\
\hline 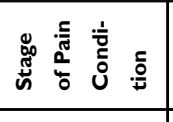 & $\begin{array}{ll}\frac{1}{\bar{c}} \\
\text { 产。 } \\
\end{array}$ \\
\hline 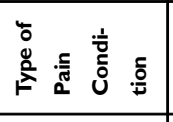 & $\frac{\hat{z}}{\mathrm{z}}$ \\
\hline 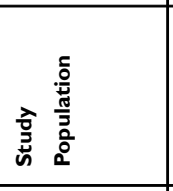 & 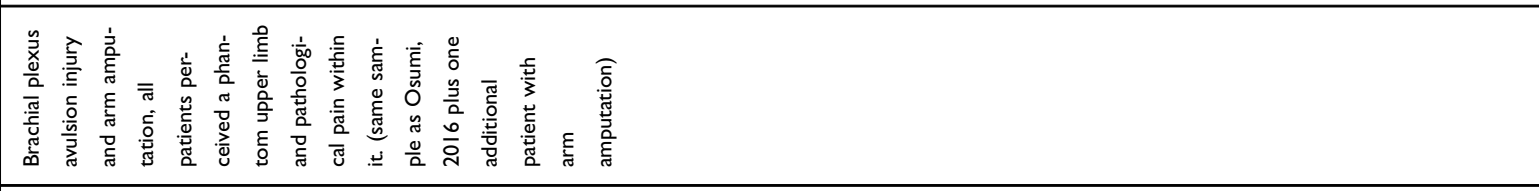 \\
\hline 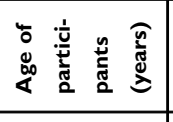 & 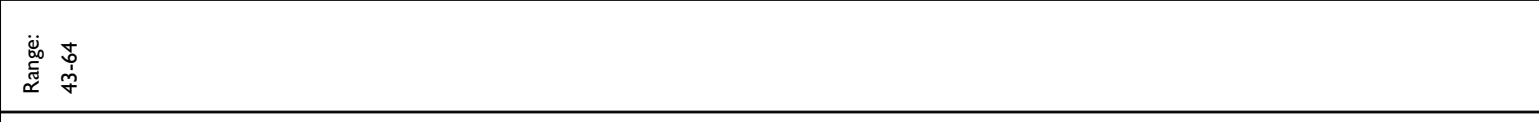 \\
\hline 迹 & $\alpha$ \\
\hline 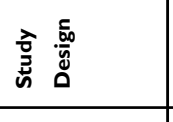 & 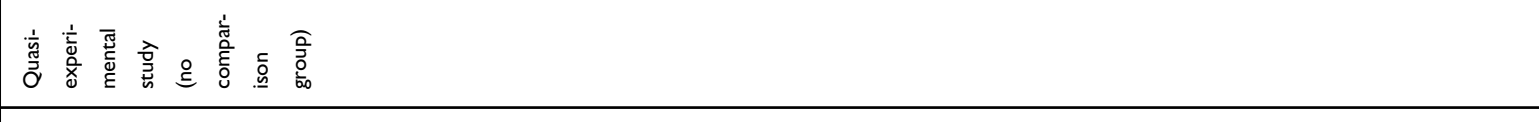 \\
\hline & 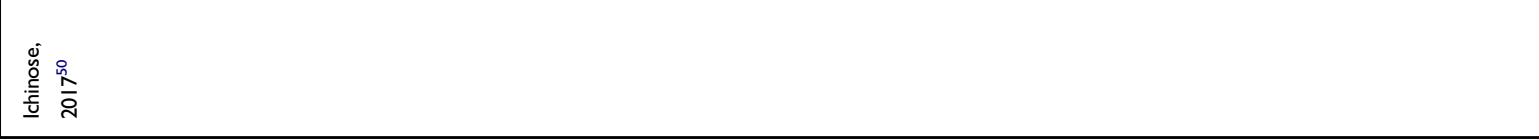 \\
\hline
\end{tabular}




\begin{tabular}{|c|c|}
\hline 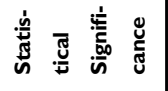 & ¿̊. \\
\hline 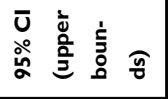 & $\stackrel{\circ}{-}$ \\
\hline 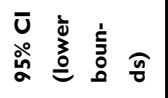 & న్ \\
\hline 立 䓀 & $\stackrel{\infty}{\circ}$ \\
\hline 今ั & 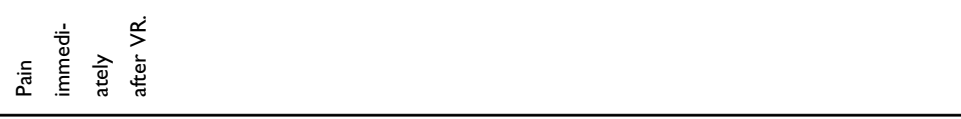 \\
\hline 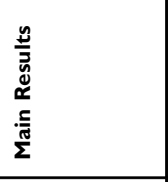 & 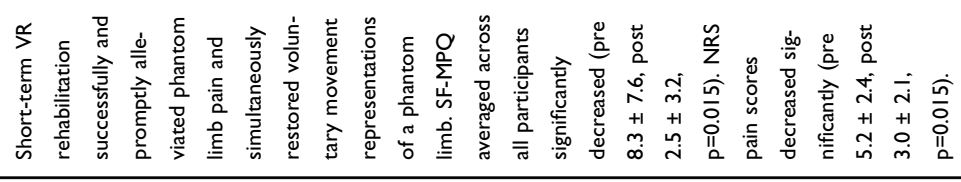 \\
\hline 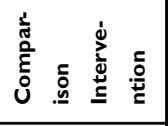 & $\stackrel{\pi}{\varepsilon}$ \\
\hline 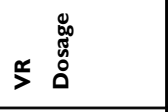 & 을 \\
\hline 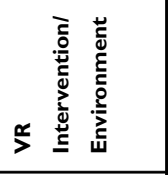 & 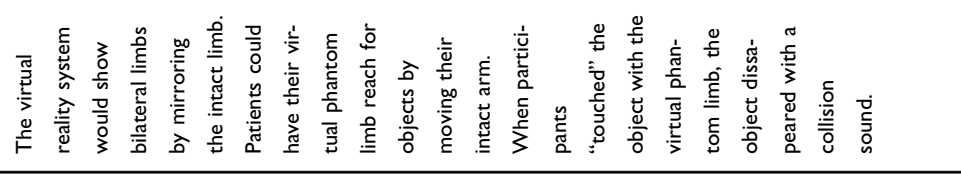 \\
\hline 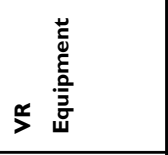 & 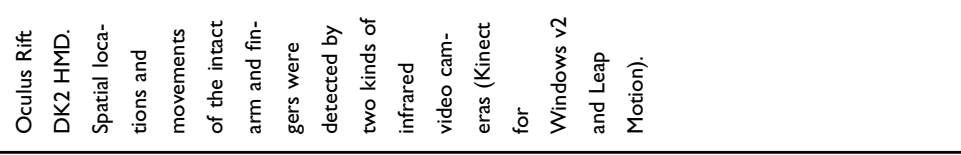 \\
\hline 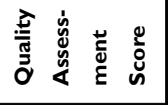 & $\stackrel{\infty}{\stackrel{\infty}{\Sigma}} \stackrel{\widehat{\Sigma}}{\leq}$ \\
\hline 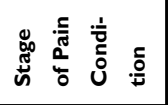 & 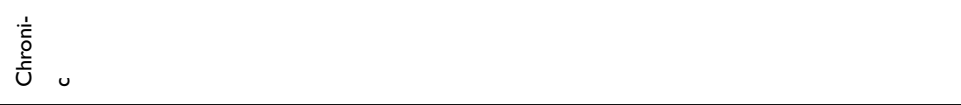 \\
\hline 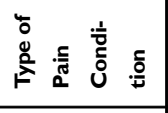 & $\hat{z}$ \\
\hline 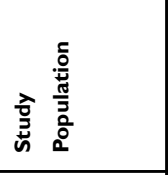 & 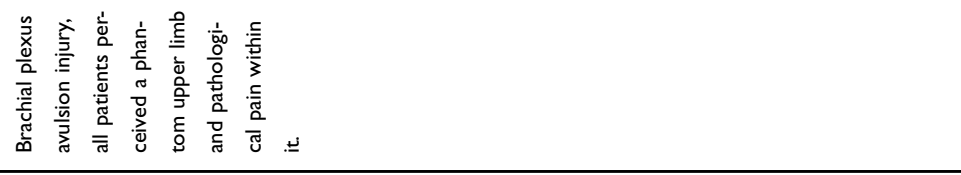 \\
\hline 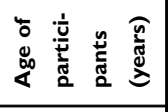 & 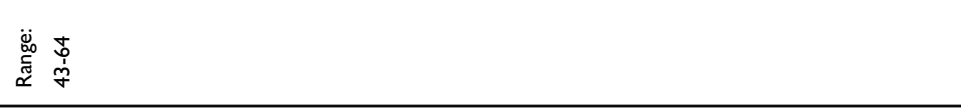 \\
\hline 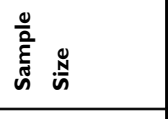 & $\infty$ \\
\hline 率离 & 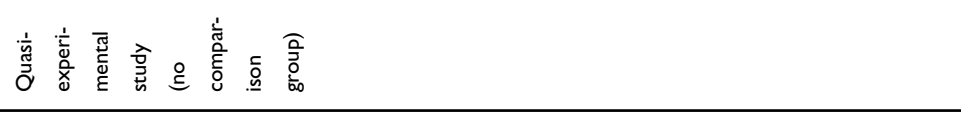 \\
\hline 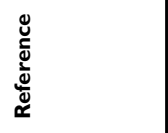 & 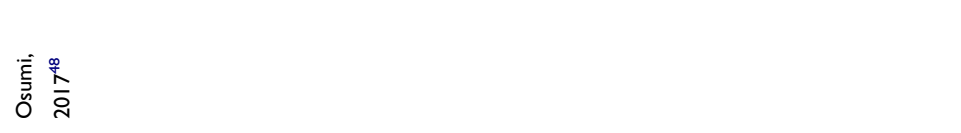 \\
\hline
\end{tabular}




\begin{tabular}{|c|c|}
\hline 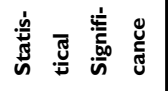 & 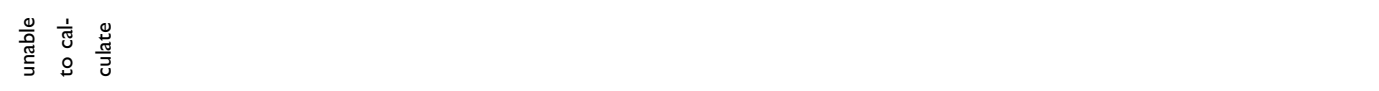 \\
\hline 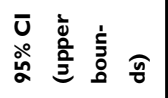 & 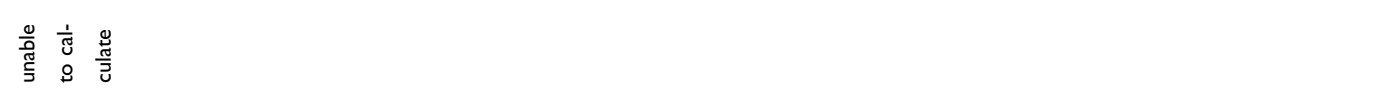 \\
\hline 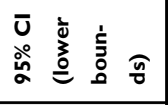 & 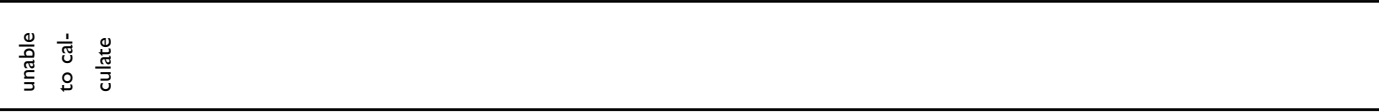 \\
\hline 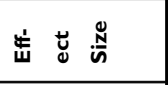 & 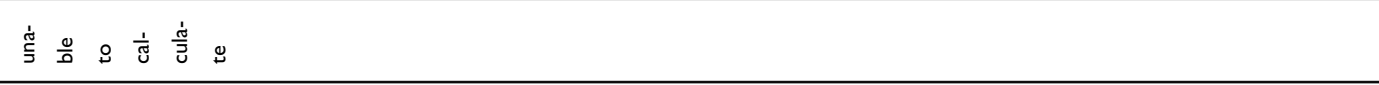 \\
\hline 乌ั & 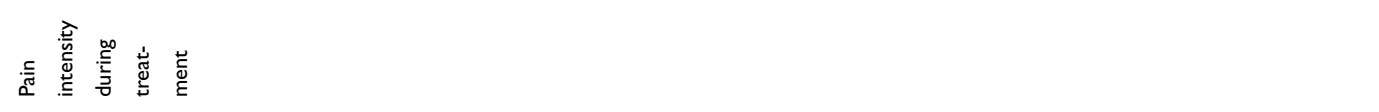 \\
\hline & 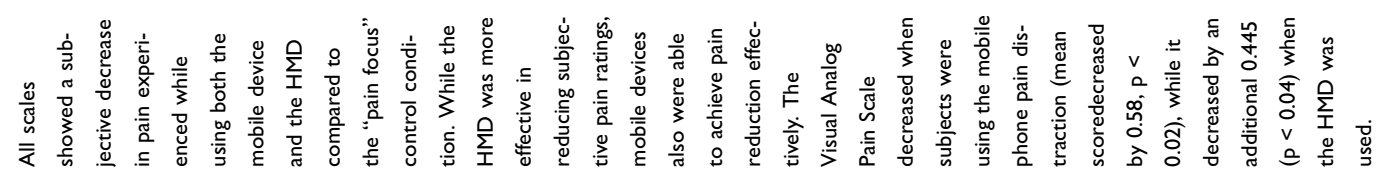 \\
\hline 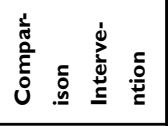 & 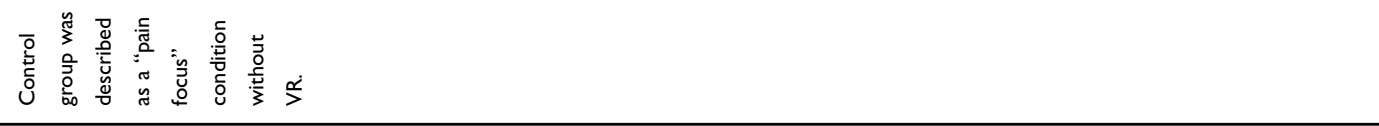 \\
\hline 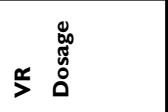 & 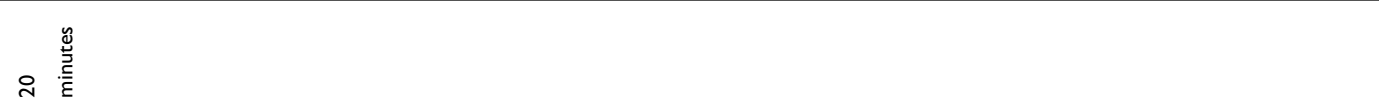 \\
\hline 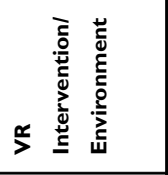 & 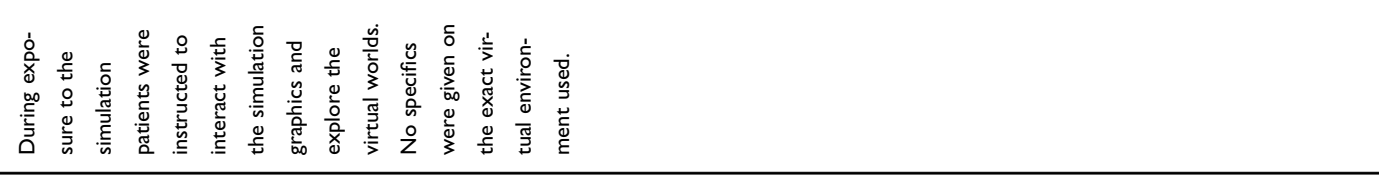 \\
\hline 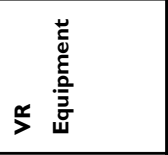 & 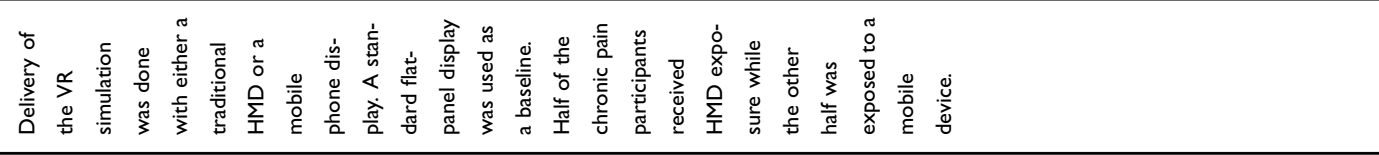 \\
\hline 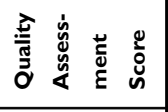 & 웄음 \\
\hline 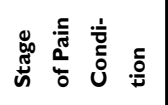 & $\frac{1}{\frac{1}{0}}$ \\
\hline 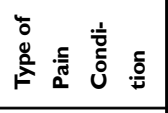 & $\hat{z}$ \\
\hline 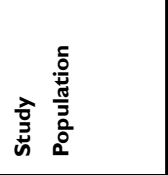 & 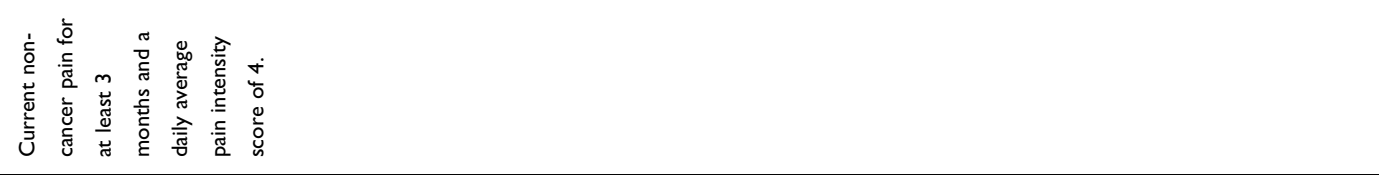 \\
\hline 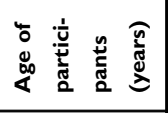 & 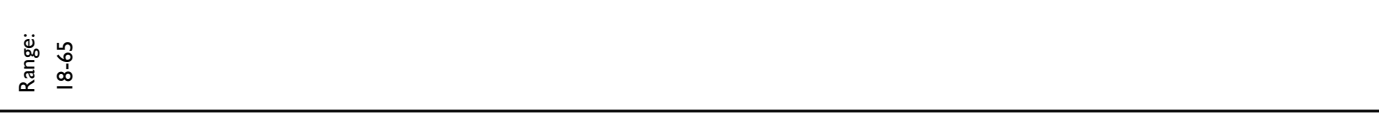 \\
\hline 递 & $\bar{m}$ \\
\hline 旁点 & $\begin{array}{l}t \\
\propto \\
\end{array}$ \\
\hline & 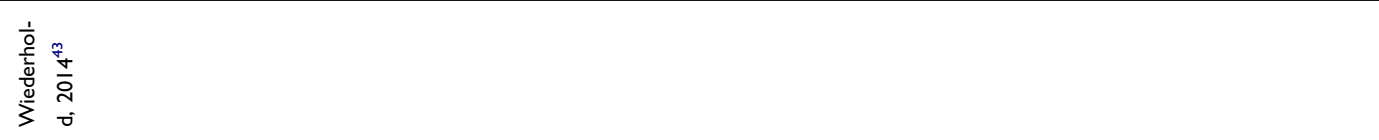 \\
\hline
\end{tabular}




\begin{tabular}{|c|c|}
\hline 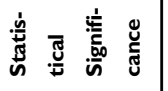 & 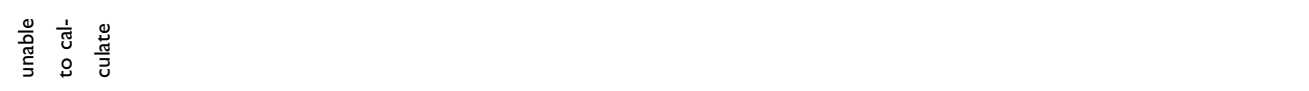 \\
\hline 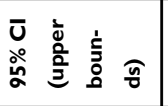 & 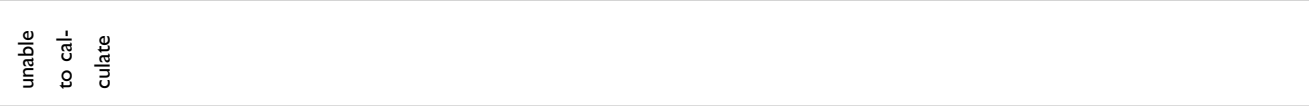 \\
\hline 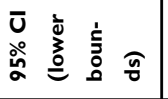 & 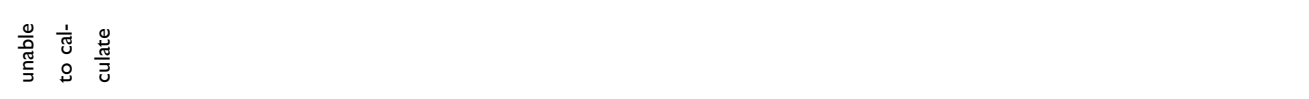 \\
\hline 岀 せัّ & 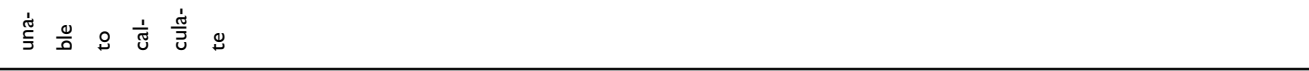 \\
\hline 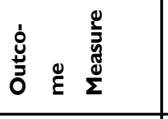 & 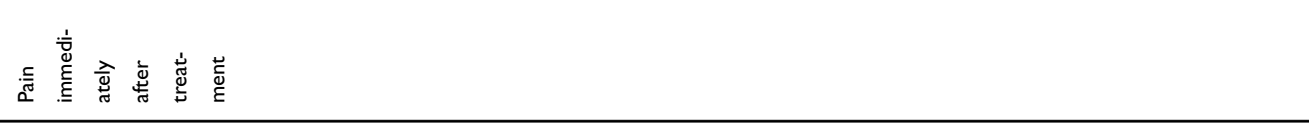 \\
\hline 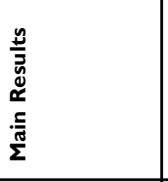 & 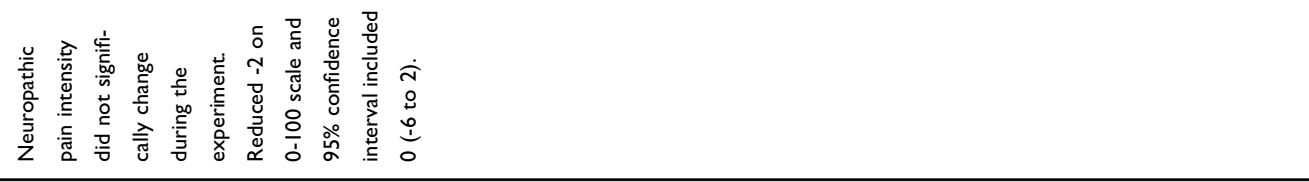 \\
\hline 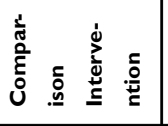 & $\frac{\pi}{\Sigma}$ \\
\hline 总 & 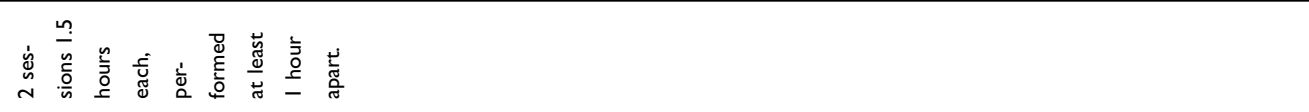 \\
\hline 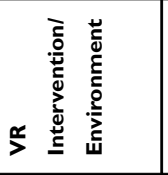 & 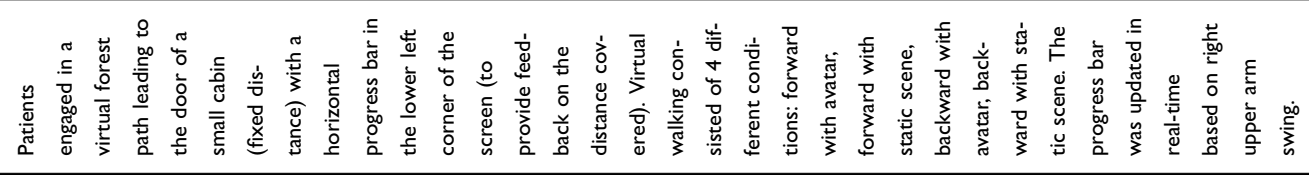 \\
\hline 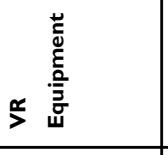 & 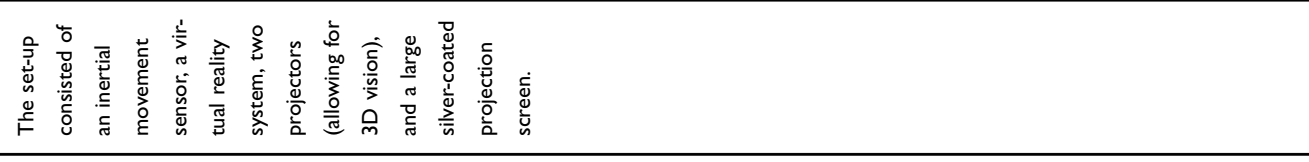 \\
\hline 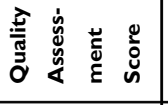 & $\stackrel{\infty}{\grave{\Sigma}} \underset{\Sigma}{\Sigma}$ \\
\hline 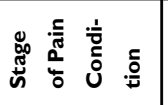 & $\begin{array}{l}\frac{1}{\overline{0}} \\
\frac{2}{2} \\
\frac{2}{U}\end{array}$ \\
\hline 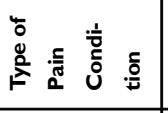 & $\hat{z}$ \\
\hline 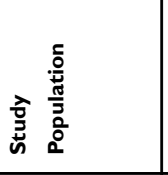 & 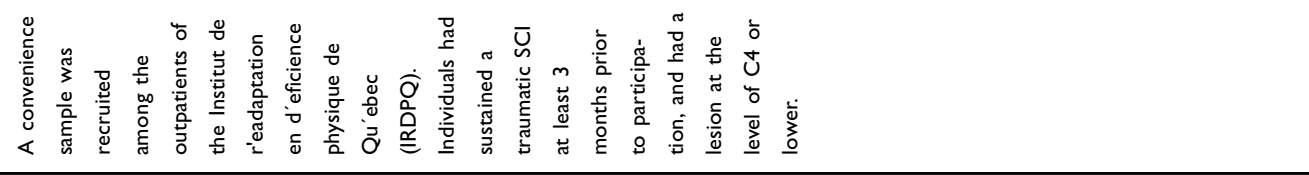 \\
\hline 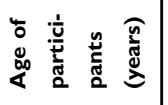 & 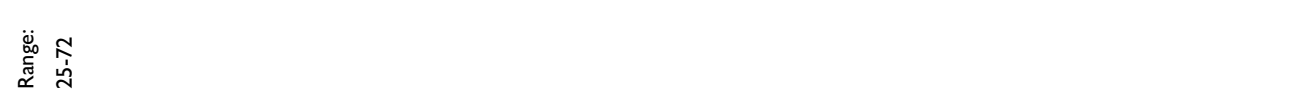 \\
\hline 造 & $\alpha$ \\
\hline 害高 & 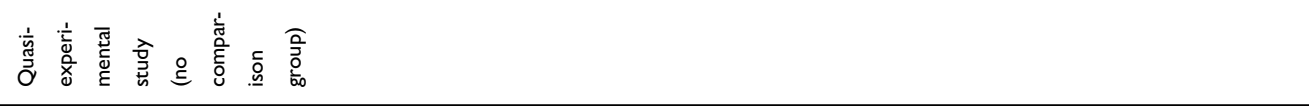 \\
\hline & 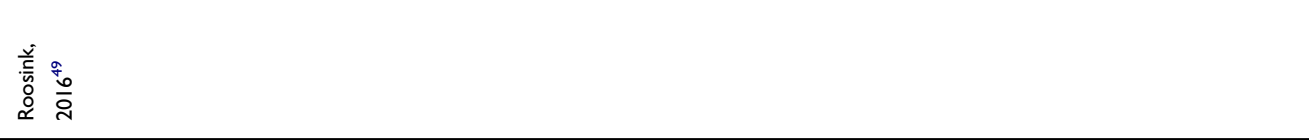 \\
\hline
\end{tabular}




\begin{tabular}{|c|c|c|}
\hline 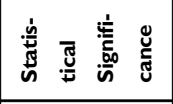 & 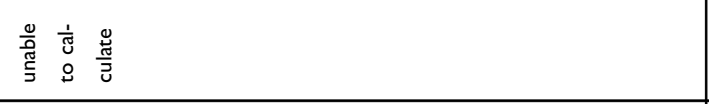 & 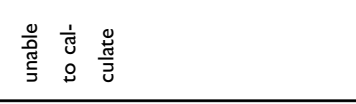 \\
\hline 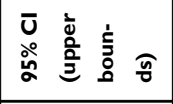 & 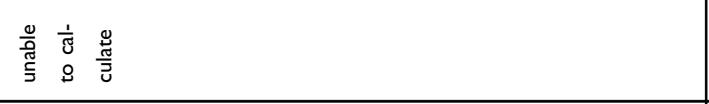 & 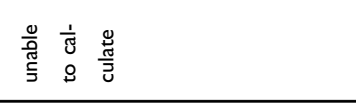 \\
\hline 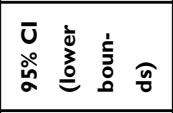 & 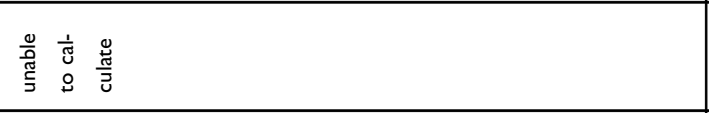 & 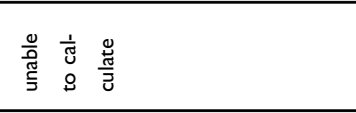 \\
\hline 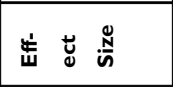 & 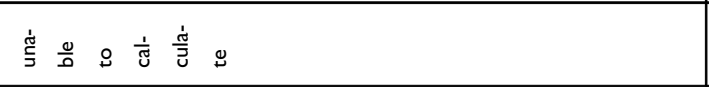 & 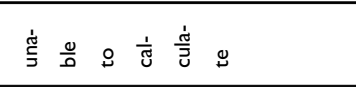 \\
\hline 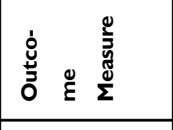 & 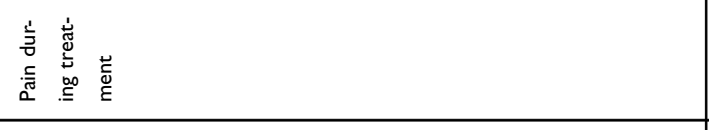 & 害离 \\
\hline 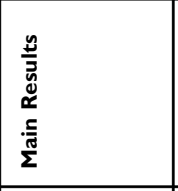 & 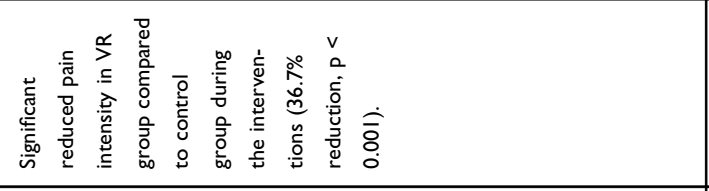 & 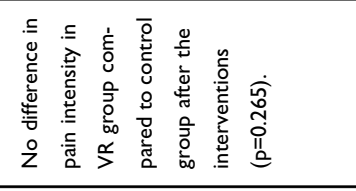 \\
\hline 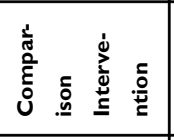 & 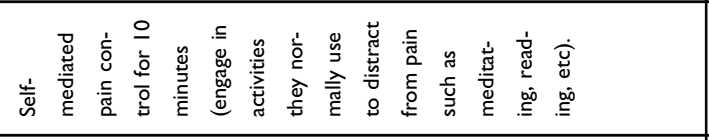 & 1 \\
\hline 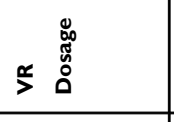 & ○蒡 & 1 \\
\hline 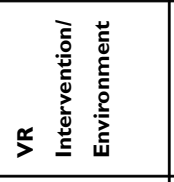 & 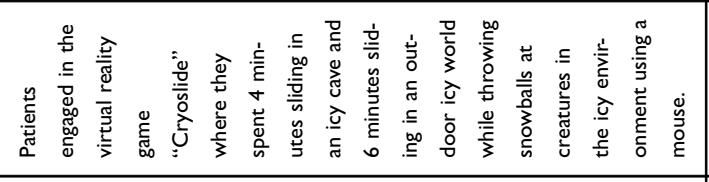 & \\
\hline 点 & 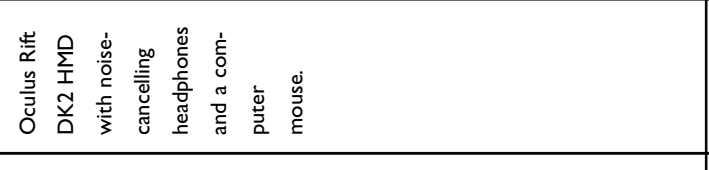 & 1 \\
\hline 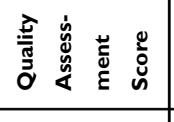 & 咅 & 1 \\
\hline 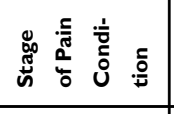 & 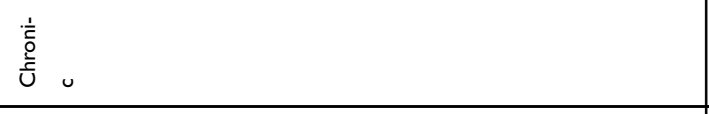 & 1 \\
\hline 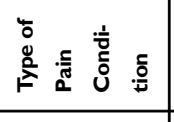 & 号 & 1 \\
\hline 毫 & 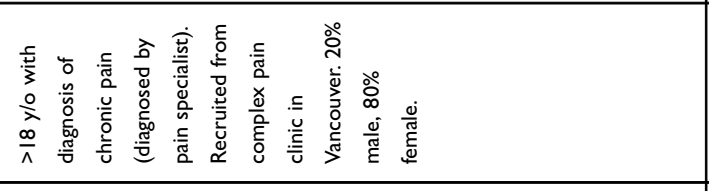 & । \\
\hline 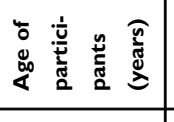 & 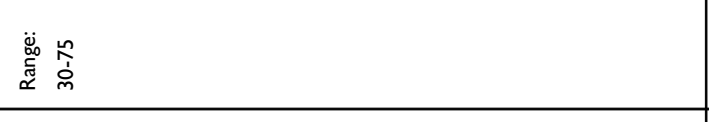 & 1 \\
\hline 造 & $\therefore$ & 1 \\
\hline 害高 & 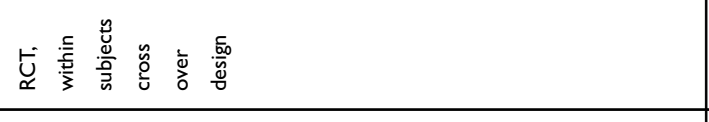 & I \\
\hline 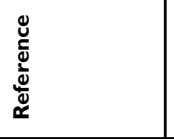 & 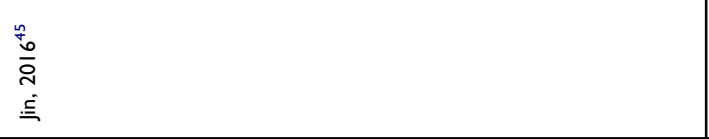 & \\
\hline
\end{tabular}




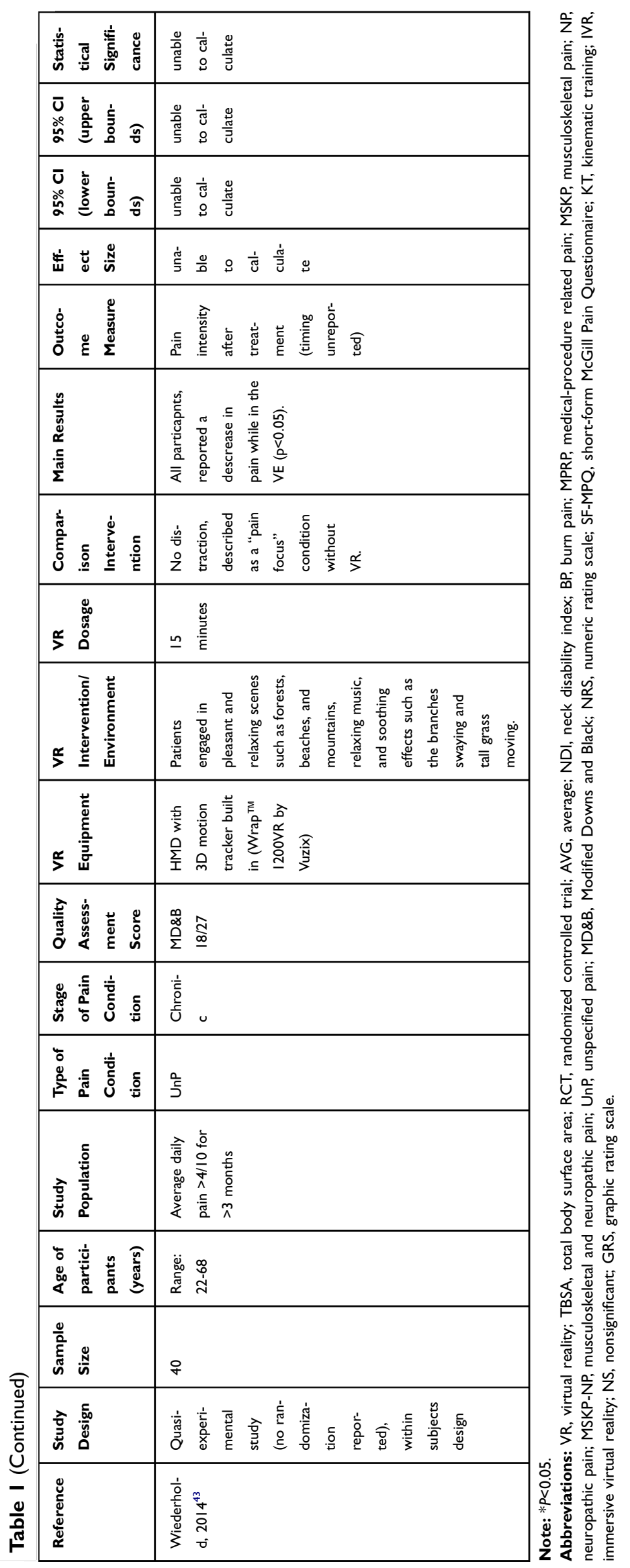




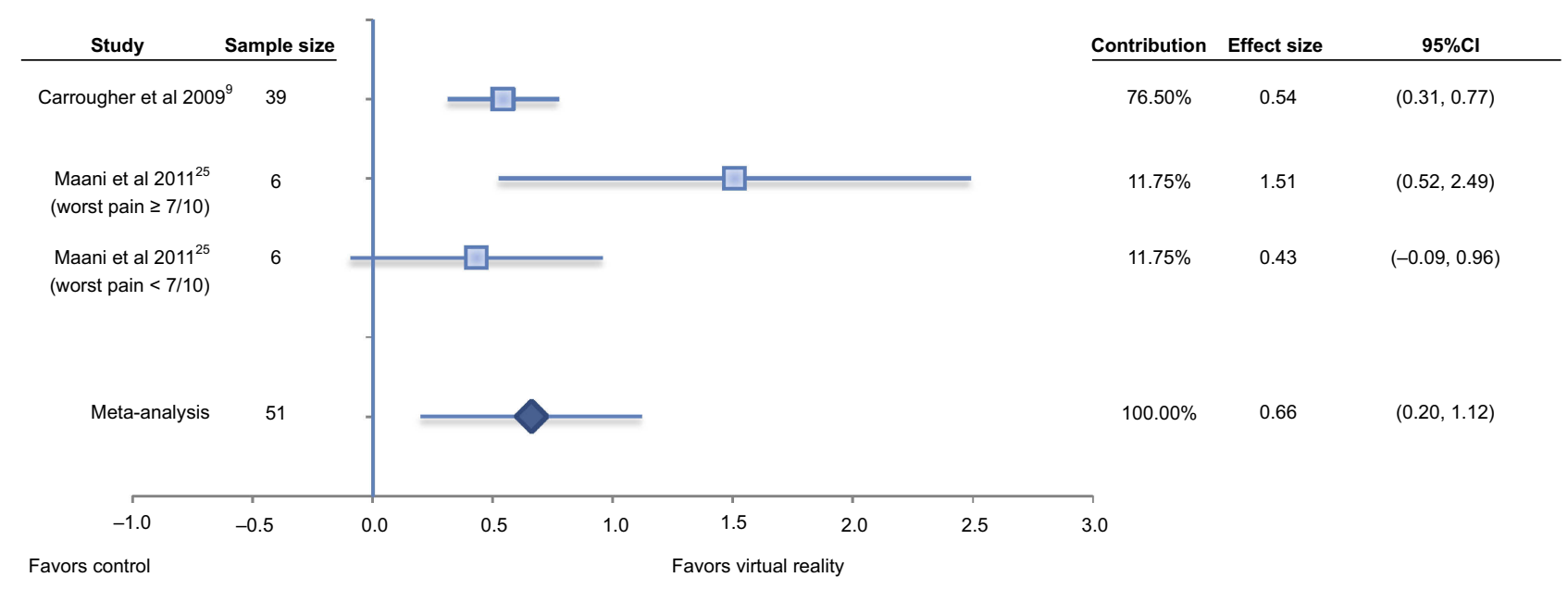

Figure 2 Effect size of virtual reality of reducing pain intensity durig burn treatments (wound management or remobilization of limbs) compared to medication alone.

The third study found VR significantly reduced the worst pain experienced during labor contractions compared to no VR (ES = unable to calculate) ${ }^{37}$ The fourth study found no significant change in either average or worst pain felt during a cystoscopy procedure with the addition of VR (ES = unable to calculate). ${ }^{36}$ However, this study found that patients reported a sense of not feeling immersed in the VR environment. Meta-analysis was not performed due to the heterogeneity of these patient populations and medical procedures. All four of these studies used a HMD for VR application and had a dosage of 10 minutes, ${ }^{37} 11$ minutes, ${ }^{35} 20$ minutes $^{34}$ or the length of the procedure, which was not specified. ${ }^{36}$ Quality assessment scores for these studies were between 6-7 and 10 on the PEDro scale and 22 and 27 on the MD\&B scale indicating high quality and low risk of bias.

\section{Impact of VR on pain experienced after procedure}

Four studies evaluated the impact of VR on pain after various MPRP. ${ }^{35,38-40}$ The first of these studies reported a reduction in pain with the addition of VR during an episiotomy repair both immediately after (ES=1.37, 95\%CI: $0.95,1.79)$ and one hour after ( $\mathrm{ES}=0.62,95 \% \mathrm{CI}: 0.34,0.90)$ the procedure compared to standard episiotomy without VR. ${ }^{35}$ The second study found significantly lower pain levels ( $\mathrm{ES}=1.87,95 \%$ CI: $1.49,2.25)$ after a dressing change for hand injury when using VR compared to the dressing change with no VR. ${ }^{38}$ The third study found no significant difference between VR after dressing change for various types of wounds (burns, ulcers, necrotizing fasciitis), compared to dressing change with no VR. ${ }^{40}$ Interestingly, we calculated a significant ES $=0.41$ (95\%CI: $0.09,0.74)$, as this statistic is less impacted by small sample size compared to $P$-values. ${ }^{41}$ Meta-analysis was not performed due to the heterogeneity of these patient populations and medical procedures. All of these studies used an HMD for VR application and had dosage from 11 minutes to approximately 30 minutes. Quality assessment scores for these studies were between 6-7 out of 10 on the PEDro scale indicating high quality and low risk of bias. The fourth study to look at pain after a medical procedure was a quasi-experimental study in patients within 24 hours of a cardiac procedure and found that the majority of patients (88\%) experienced less pain immediately after a 30-minute application of VR with an HMD. ${ }^{39}$ The quality assessment score for this study was 20/27 on the MD\&B scale indicating low risk of bias.

\section{Chronic pain conditions Impact of VR on pain experienced during VR exposure}

One study examined the effect of various forms of VR manipulation on chronic neck pain (MSKP) during the VR exposure. ${ }^{42}$ Specifically, they compared VR with active neck rotation motions to the onset of pain such that the VR environment either matched, underepresented (by $20 \%$ ), or overepresented (by 20\%) the actual neck motion. They did not compare to a nonR condition. They found that pain free range of motion increased with the VR experience that underrepresented the actual neck motion but that there was no difference in neck pain intensity experienced between the three conditions (ES = unable to calculate). This study used an HMD with a single rotation to each side for the three conditions for a total of six motions with VR. The PEDro score for this study was 8/ 10 indicating high quality and low risk of bias. 
One study examined chronic NP with 20 minutes exposure to VR using an HMD or a mobile phone display compared to a control condition described as "pain focus" without VR. ${ }^{43}$ This study found a significant reduction in pain during the VR exposure for both the HMD and mobile device compared to the control condition ( $\mathrm{ES}=$ unable to calculate). The HMD led to a greater reduction in pain than the mobile device. The PEDro score for this study was $5 / 10$ indicating a fair quality rating.

Another study examined a 5-minute exposure to VR using an HMD in patients with chronic MSKP-NP conditions but did not compare to a comparison group (quasiexperimental study). ${ }^{44}$ This study found a significant decrease in pain within this group during the VR exposure by an average of $60 \%(P<0.001)$. The MD\&B score was $18 / 27$ indicating low risk of bias.

Finally, another study examined patients with UnP conditions. ${ }^{45}$ In this study, they compared a 10-minute exposure of VR using an HMD to 10 minutes of selfmediated pain control where patients were instructed to engage in activities that they would normally use to distract them from their pain. They found that VR reduced pain intensity during the VR exposure by $36.7 \%(P<0.001)$ (ES = unable to calculate). The PEDro score for this study was $5 / 10$ indicating a fair quality rating.

\section{Impact of VR on pain experienced after VR exposure}

Two studies examined pain intensity after VR exposure in patients with MSKP. ${ }^{46,47}$ The first of these studies projected a VR environment onto a screen in front of a treadmill where the participant walked at a self-selected speed for 12 bouts of 2 minutes with the audio feedback being either absent or present with a $75 \%, 100 \%$ or $125 \%$ match of their walking cadence. ${ }^{46}$ They did not compare to a nonVR condition. They found that the VR experience with audio feedback provided at $125 \%$ of their baseline cadence led to an increase walking speed but that there was no significant change in pain intensity immediately after the VR exposures ( $E S=$ unable to calculate). The PEDro score for this study was $5 / 10$ indicating a fair quality rating. The second study compared VR using HMD for 4-6 sessions of 30 minutes (over 5 weeks) in conjunction with kinematic training (KT) of active head and neck movements compared to the KT without VR. ${ }^{47}$ They found that the VR group had a significant reduction in pain immediately after the VR exposure $(P<0.05)(\mathrm{ES}=0.65,95 \% \mathrm{CI}: 0.25,1.05)$ but that this was not maintained at a 3-month follow-up (ES $=0.51,95 \% \mathrm{CI}$ : $0.14,0.88$ ). They also noted significant reductions in the Neck Disability Index (NDI) both immediately after VR exposure and at the 3-month follow-up ( $P<0.01$ for both). The PEDro score for this study was $7 /$ 10 indicating high quality and low risk of bias.

Three studies examined pain intensity after VR exposure in NP conditions using a quasi-experimental design. ${ }^{48-50}$ The first of these studies evaluated a 10-minute VR exposure using HMD in patients with brachial plexus avulsion injuries that resulted in phantom limb pain. ${ }^{48}$ A subsequent paper by the same authors reported on this same sample with the addition of one additional patient with an arm amputation under slightly different VR testing conditions. ${ }^{50}$ Osumi et al ${ }^{48}$ found that pain was significantly reduced immediately after 10 minutes of VR exposure $(P=0.015)$. Similarly, Ichinose et $\mathrm{al}^{50}$ found that pain was significantly reduced immediately after 15 minutes of VR exposure that included tactile stimulation on the patient's cheek that coincided with a task within the VR environment $(P=0.004)$. The MD\&B score was $17 / 27$ for both studies indicating low risk of bias. The third study evaluated twosessions of 1.5 hours ( $\geq 1$ hour apart) of a VR environment projected onto two screens in front of patients with a spinal cord injury (C4 or lower) at least 3 months prior. $^{49}$ This study found no significant difference in pain reported immediately after the VR exposure. The MD\&B score was 17/27 indicating low risk of bias.

Another study examined a 5-minute exposure to VR using an HMD in patients with chronic MSKP-NP conditions but did not compare to a comparison group (quasiexperimental study). ${ }^{44}$ This study found a significant decrease in pain within this group immediately after the VR exposure by an average of $33 \%(P<0.001)$. The MD\&B score was 18/27 indicating low risk of bias.

Two studies examined the impact of VR on pain intensity after VR exposure in patients with chronic UnP conditions. $^{43,45}$ The first study compared a 10-minute exposure of VR using an HMD to a self-mediated pain control where patients were instructed to engage in activities that they would normally use to distract from their pain for 10 minutes without VR. ${ }^{45}$ They found no difference in pain intensity between groups within 10 minutes of completion of the VR exposure $(P=0.265)(\mathrm{ES}=$ unable to calculate). The PEDro score for this study was $5 / 10$ indicating a fair quality rating. The other study evaluated 15 minutes exposure to VR using an HMD compared to a control condition described as "pain focus" without VR (within subjects design, no randomization reported). ${ }^{43}$ They found a significant reduction in pain intensity after VR exposure (time frame not reported) $(P<0.05)(\mathrm{ES}=$ 
unable to calculate). The MD\&B score was 18/27 indicating low risk of bias.

\section{Discussion}

The majority of the acute pain studies showed that VR is an effective tool in reducing acute pain experienced during various medical procedures (labor contractions, episiotomy repair, periodontal procedures) or burn-related pain (wound debridement or remobilization of range of motion for joints limited by burns). Only one study of acute pain found no difference in pain experienced during a cystoscopy procedure with the addition of VR, however, the authors reported the patients did not feel immersed in the VR environment. ${ }^{36}$

In comparison, three of the four acute pain studies that looked at pain experienced after various medical procedures (episiotomy repair, dressing changes for hand injuries, and cardiac procedures) found a significant reduction in pain with the addition of VR. One study did not find a significant difference in pain levels after wound care with VR compared to no VR. However, we calculated a moderate effect size in favor of VR, indicating that this study may have been underpowered to detect this level of effect. All of these studies were rated as high quality and low risk of bias. These findings suggests that there is significant evidence to support the use of VR to reduce the severity of pain experienced both during and after many medical procedures. The magnitude of pain reduction varies depending on patient population, pain condition, and timing of measurement.

The findings for the chronic pain studies are less consistent. The two experimental trials in patients with chronic NP or UnP that compared to a nonVR comparison group (a "pain focus" condition or a self-mediated pain control session, respectively) found that pain intensity was significantly reduced during the VR exposure. ${ }^{43,45}$ The quality assessment for these two studies was only fair indicating some potential risk of bias in the study design. Further support for reductions in pain intensity during VR exposure in patients with chronic pain comes from a study of patients with MSKP-NP. ${ }^{44}$ In contrast, another study looking at various forms of VR experiences in MSKP found no difference in neck pain during three versions of VR exposure. ${ }^{42}$ Although the two aforementioned studies had low risk of bias, they did not compare to a nonVR condition which limits the ability to evaluate the impact of VR compared to other forms of intervention for chronic pain.
Two experimental trials in patients with either chronic $\mathrm{MSKP}^{47}$ or chronic $\mathrm{UnP}^{43}$ found reduced pain after VR exposure compared to nonVR comparisons (kinematic training of active head and neck movements or a selfmediated pain control session, respectively). The former study found that reductions in pain level were not maintained at 3 months after VR exposure. In contrast, one study found no significant difference in pain intensity within 10 minutes after VR exposure compared to a "pain focus" condition in patients with UnP. ${ }^{45}$ A similar pattern was noted from quasi-experimental studies, such that three studies found a reduction of pain after VR exposure in patients with NP or MSK-NP and one study found no difference in patients with NP. Lastly, another study looking at various forms of VR experiences in MSKP found no difference in pain experienced immediately after VR exposure, however, they did not compare to a nonVR condition, which limits the ability to evaluate the impact of VR compared to other forms of intervention for chronic pain. ${ }^{46}$

All chronic pain studies had fair to high quality assessment ratings. The findings of the chronic pain studies suggest that there is potential for VR to reduce pain during VR exposure in patients with chronic pain. However, there are inconsistent findings for the use of VR to reduce pain after VR exposure in patients with chronic pain. Therefore, it may be speculated that although VR may effectively reduce chronic pain intensity during and possibly immediately after the VR exposure, there is unlikely to be a lasting analgesic effect in patients with chronic pain conditions with the treatment protocols tested to date.

One possibility for the variance found in our chronic pain results is the dosage of VR intervention. For example, when patients underwent a single 10minute bout of VR, the VR group had no significant reduction in their pain. ${ }^{45}$ However, when patients underwent 4-6×30-minute sessions over a 5-week period, the VR group had a significant reduction in pain immediately after VR exposure. ${ }^{47}$ This may indicate the need for a larger dose of VR therapy to impact pain for people with chronic pain conditions, but requires further research.

A second possibility may be related to the type of VR equipment used. The majority of studies used a form of glasses/goggles or other forms of HMD, while two studies used projectors. The two studies that used projectors did not support the use of VR to alter chronic pain. Therefore, it may be possible that the use of an HMD allows for a 
greater level of immersion and thus more distraction from the user's pain than a projector.

Our findings are consistent with previous systematic reviews, which have found VR to be effective in reducing acute pain. ${ }^{18,19,21,51}$ However, our systematic review includes more research on pain intensity and makes a direct comparison between changes in acute and chronic pain. One systematic review concluded that VR distraction was ineffective in reducing chronic pain, however, this conclusion was based on only one study. ${ }^{19}$ We were able to compare 10 chronic pain articles and found new evidence to suggest that VR intervention has the potential to significantly reduce chronic pain during VR intervention. The most recent systematic review ${ }^{19}$ did not provide quality assessment scores for included articles, which limits the reader's ability to assess the validity of their findings.

This systematic review was focused on the effects of VR intervention on reducing pain intensity, but other studies have looked at the effects of VR on functional outcomes. For example, Sarig Bahat et $\mathrm{al}^{47}$ measured the impact of chronic neck pain on functional performance using the NDI and found that the VR group had positive changes in NDI scores for 3 months postintervention compared to the control group. It will be important to further examine the effect of VR on function in future research.

One strength of our review was the broad search strategy including three databases and searching other systematic reviews on the same topic for any studies that met our criteria. Previous studies ${ }^{19,27}$ have focused on experimental pain or have focused solely on pediatric populations. We excluded experimental pain to promote clinical relevance of our findings and excluded pediatric populations to improve our study's applicability to adults.

There was significant heterogeneity in study population and pain conditions, which poses a limitation for this systematic review. Additionally, our review is limited by the variety of the VR equipment used and the dosage of VR intervention in the included studies. One other limitation is that we included articles written in English only.

As VR continues to emerge as an adjunctive pain reduction treatment, it is important to consider the cost, feasibility and accessibility of VR equipment. The VR equipment in this review ranged from a high cost multiple-projector set-up to a low-cost commercially available system and the VR market is predicted to grow $29.2 \%$ by $2020 .^{52}$ With this evolving market, it is an ethical consideration to ensure that access to VR treatment does not become cost-prohibitive and that application of this intervention is supported by the literature. Future studies should continue to examine the cost-effectiveness of VR as a pain management tool, and if it is feasible to use lowcost VR systems to reduce the financial burden on the health-care system and improve access.

In addition, this review highlights the need for high quality randomized clinical trials for VR in patients with chronic pain conditions. It is recommended that studies evaluate appropriate dosage of VR exposure compared to other pain analgesia therapies (ie pain medications, visual illusions, mirror box therapy, etc.) for both short and longterm outcomes that are meaningful to the patient (including pain reduction, function and quality of life). Moreover, future studies should examine the effects of VR on pain control in different clinical settings (including intensive care, acute care and outpatient settings).

One additional recommendation comes from the considerable variability in the definition of immersive VR across studies. A possible solution is to provide a standardized measurement of how immersed participants feel when undergoing VR intervention. Some of the studies included in our review used subjective measures of immersiveness to quantify their experience of immersion. For example, participants rated their sense of presence in the virtual environment on a VAS scale from, "I did not feel like I went inside at all," to, "I went completely into the computer-generated world." ${ }^{\prime}$ However, we believe a more comprehensive tool is warranted due to the complexity of immersion. One of our included studies ${ }^{12}$ used the Presence Questionnaire (PQ) developed by Witmer and Singer. ${ }^{51}$ The PQ is a 24-question, valid and reliable measure of presence in a virtual world. ${ }^{53}$ We recommend researchers consider the PQ for its ability to capture multiple dimensions of the patient's experience.

Finally, it may also be imperative to determine if there is a clinical difference between "immersion" and "presence." "Presence" may be defined as the subjective illusion in the participant's mind that he or she has gone inside the virtual world. ${ }^{19,51}$ For example, the person can receive sensory feedback from the virtual world, but may not be "present" if they are not cognitively engaged in it. Previous studies have attempted to explain VR's effect on pain, noting that decreased activation of pain processing areas in the brain may be responsible for subjective pain reduction with VR intervention. ${ }^{7,51,54}$ A shift in cognitive demand can lead to distraction from pain, and being "present" in the virtual environment can theoretically stimulate this shift. Even if someone is "immersed" in a virtual world, they may not be 
"present" in it. We recommend further research to determine if "immersion" and "presence" are conceptually different, and if so what impact they have on VR in reducing pain.

\section{Conclusion}

This study provides an updated systematic literature review showing the effects of VR on both acute and chronic pain. We conclude that VR is an effective tool in reducing acute pain both during and after a VR intervention, and that VR is especially effective in mitigating MPRP. The evidence for chronic pain relief with VR suggests that chronic pain may be reduced while the patient is immersed in the VR environment, but with minimal long-term carryover effect beyond the immediate postVR exposure time frame. Further research is needed to assess the extent to which one needs to be immersed and present in a virtual environment in order to reduce pain, and the dosage necessary to maintain pain reductions in chronic pain over time. Clinicians should consider immersive VR therapies as an adjunct to ${ }^{23}$ standard care to help reduce acute pain and potentially for chronic pain conditions.

\section{Acknowledgments}

The authors are grateful to Dr Jason Hardage for his assistance with our review process and to Meira Prescher, Ping Zhang, Eric Kwan, and AJ Pluss for their thorough peer review. No sources of funding were used to create this systematic review.

\section{Disclosure}

The authors report no conflicts of interest in this work.

\section{References}

1. Rasu RS, Vouthy K, Crowl AN, et al. Cost of pain medication to treat adult patients with nonmalignant chronic pain in the United States. $J$ Manag Care Spec Pharm. 2014;20(9):921-928. doi:10.18553/ jmcp.2014.20.9.921

2. Teater D The psychological and physical side effects of pain medications. 2014. Available from: http://safety.nsc.org/sideeffects. Accessed September 25, 2018.

3. Sutherland S Meeting report: virtual reality pain 2016 | pain research forum. 2016. Available from: https://www.painresearchforum.org/ news/74439-meeting-report-virtual-reality-pain-2016. Accessed September 25, 2018.

4. Greenleaf $\mathrm{W}$ How virtual and augmented reality will change the way we shop | techno FAQ. Available from: https://www.slideshare.net/waltergreen leaf/how-virtual-and-augmented-reality-will-transform-healthcare-august2017-presentation?qid=16451079-9e92-44a7-9baf-1e1d237c8575\&v= $\& b=\&$ from_search $=1$. Accessed September 25, 2018.

5. Hoffman HG, Doctor JN, Patterson DR, Carrougher GJ, Furness TA. Virtual reality as an adjunctive pain control during burn wound care in adolescent patients. Pain. 2000;85(1-2):305-309.
6. Hoffman HG, Patterson DR, Carrougher GJ, Sharar SR. Effectiveness of virtual reality-based pain control with multiple treatments. Clin J Pain. 2001;17(3):229-235. doi:10.18553/jmcp.2014.20.9.921

7. Hoffman HG, Seibel EJ, Richards TL, Furness TA, Patterson DR, Sharar SR. Virtual reality helmet display quality influences the magnitude of virtual reality analgesia. J Pain. 2006;7(11):843-850. doi:10.1016/j.jpain.2006.04.006

8. Hoffman HG, Richards TL, Van Oostrom T, et al. The analgesic effects of opioids and immersive virtual reality distraction: evidence from subjective and functional brain imaging assessments. Anesth Analg. 2007;105(6):1776-1783. doi:10.1213/01.ane.0000270205. 45146.db

9. Carrougher GJ, Hoffman HG, Nakamura D, et al. The effect of virtual reality on pain and range of motion in adults with burn injuries. J Burn Care Res. 2009;30(5):785-791. doi:10.1097/ BCR.0b013e3181b485d3

10. Hoffman HG, Chambers GT, Meyer WJ, et al. Virtual reality as an adjunctive non-pharmacologic analgesic for acute burn pain during medical procedures. Ann Behav Med. 2011;41(2):183-191. doi:10.1007/s12160-010-9248-7

11. Schmitt YS, Hoffman HG, Blough DK, et al. A randomized, controlled trial of immersive virtual reality analgesia, during physical therapy for pediatric burns. Burns. 2011;37(1):61-68. doi:10.1016/j. burns.2010.07.007

12. Schneider SM, Hood LE. Virtual reality: a distraction intervention for chemotherapy. Oncol Nurs Forum. 2007;34(1):39-46. doi:10.1188/ 07.ONF.39-46

13. Schneider SM, Ellis M, Coombs WT, Shonkwiler EL, Folsom LC. Virtual reality intervention for older women with breast cancer. Cyberpsychol Behav. 2003;6(3):301-307. doi:10.1089/ 109493103322011605

14. Windich-Biermeier A, Sjoberg I, Dale JC, Eshelman D, Guzzetta CE. Effects of distraction on pain, fear, and distress during venous port access and venipuncture in children and adolescents with cancer. $J$ Pediatr Oncol Nurs. 2007;24(1):8-19. doi:10.1177/ 1043454206296018

15. Sharar SR, Miller W, Teeley A, et al. Applications of virtual reality for pain management in burn-injured patients. Expert Rev Neurother. 2008;8(11):1667-1674. doi:10.1586/14737175.8.11.1667

16. Miller K, Rodger S, Bucolo S, Greer R, Kimble RM. Multi-modal distraction. Using technology to combat pain in young children with burn injuries. Burns. 2010;36(5):647-658. doi:10.1016/j. burns.2009.06.199

17. Garrett B, Taverner T, Masinde W, Gromala D, Shaw C, Negraeff M. A rapid evidence assessment of immersive virtual reality as an adjunct therapy in acute pain management in clinical practice. Clin J Pain. 2014;30(12):1089-1098. doi:10.1097/AJP.0000000000000064

18. Shahrbanian S, Ma X, Korner-Bitensky N, Simmonds MJ. Scientific evidence for the effectiveness of virtual reality for pain reduction in adults with acute or chronic pain. Stud Health Technol Inform. 2009;144:40-43.

19. Malloy KM, Milling LS. The effectiveness of virtual reality distraction for pain reduction: a systematic review. Clin Psychol Rev. 2010;30(8):1011-1018. doi:10.1016/j.cpr.2010.07.001

20. Macedonio MF, Parsons TD, DiGiuseppe RA, Weiderhold BK, Rizzo AA. Immersiveness and physiological arousal within panoramic video-based virtual reality. Cyberpsychol Behav. 2007;10(4):508515. doi: $10.1089 / \mathrm{cpb} .2007 .9997$

21. Chan E, Foster S, Sambell R, Leong P. Clinical efficacy of virtual reality for acute procedural pain management: a systematic review and meta-analysis. PLoS One. 2018;13(7):e0200987. doi:10.1371/ journal.pone.0200987

22. Gupta A, Scott K, Dukewich M. Innovative technology using virtual reality in the treatment of pain: does it reduce pain via distraction, or is there more to it? Pain Med. 2018;19(1):151-159. doi:10.1093/pm/ pnx109 
23. Treede R-D, Rief W, Barke A, et al. A classification of chronic pain for ICD-11. Pain. 2015;156(6):1. doi:10.1097/j. pain. 0000000000000160

24. Boesch E, Bellan V, Moseley GL, Stanton TR. The effect of bodily illusions on clinical pain. Pain. 2016;157(3):516-529. doi:10.1097/j. pain. 0000000000000423

25. Maani CV, Hoffman HG, Morrow M, et al. Virtual reality pain control during burn wound debridement of combat-related burn injuries using robot-like arm mounted VR goggles. $J$ Trauma. 2011;71(1 Suppl):S125-S130. doi:10.1097/TA.0b013e31822192e2

26. Hoffman HG, Patterson DR, Carrougher GJ. Use of virtual reality for adjunctive treatment of adult burn pain during physical therapy: a controlled study. Clin J Pain. 2000;16(3):244-250.

27. Wender R, Hoffman HG, Hunner HH, Seibel EJ, Patterson DR, Sharar SR. Interactivity influences the magnitude of virtual reality analgesia. $J$ Cyber Ther Rehabil. 2009;2(1):27-33.

28. Neyeloff JL, Fuchs SC, Moreira LB. Meta-analyses and Forest plots using a microsoft excel spreadsheet: step-by-step guide focusing on descriptive data analysis. BMC Res Notes. 2012;5(1):52. doi:10.1186/ 1756-0500-5-52

29. de Morton NA. The PEDro scale is a valid measure of the methodological quality of clinical trials: a demographic study. Aust $J$ Physiother. 2009;55(2):129-133.

30. Downs SH, Black N. The feasibility of creating a checklist for the assessment of the methodological quality both of randomised and non-randomised studies of health care interventions. J Epidemiol Community Health. 1998;52(6):377-384. doi:10.1136/jech.52.6.377

31. Teasell RW, Foley NC, Bhogal SK, Speechley MR. An evidencebased review of stroke rehabilitation. Top Stroke Rehabil. 2003;10 (1):29-58. doi:10.1310/8YNA-1YHK-YMHB-XTE1

32. Abou El Fadl R, Blair M, Hassounah S. Integrating maternal and children's oral health promotion into nursing and midwifery practicea systematic seview. PLoS One. 2016;11(11):e0166760. doi:10.1371/ journal.pone. 0166760

33. Morris LD, Louw QA, Crous LC. Feasibility and potential effect of a low-cost virtual reality system on reducing pain and anxiety in adult burn injury patients during physiotherapy in a developing country. Burns. 2010;36(5):659-664. doi:10.1016/j.burns.2009.09.005

34. Furman E, Jasinevicius TR, Bissada NF, Victoroff KZ, Skillicorn R, Buchner M. Virtual reality distraction for pain control during periodontal scaling and root planing procedures. $J$ Am Dent Assoc. 2009;140(12):1508-1516.

35. JahaniShoorab N, Ebrahimzadeh Zagami S, Nahvi A, et al. The effect of virtual reality on pain in primiparity women during episiotomy repair: a randomize clinical trial. Iran J Med Sci. 2015;40(3):219224.

36. Walker MR, Kallingal GJS, Musser JE, Folen R, Stetz MC, Clark JY. Treatment efficacy of virtual reality distraction in the reduction of pain and anxiety during cystoscopy. Mil Med. 2014;179(8):891-896. doi:10.7205/MILMED-D-13-00343

37. Frey DP, Bauer ME, Bell CL, et al. Virtual reality analgesia in labor. Anesth Analg. 2018:1. doi:10.1213/ANE.0000000000003649

38. Guo C, Deng H, Yang J. Effect of virtual reality distraction on pain among patients with hand injury undergoing dressing change. $J$ Clin Nurs. 2015;24(1-2):115-120. doi:10.1111/jocn.12626

39. Mosso-Vázquez JL, Gao K, Wiederhold BK, Wiederhold MD. Virtual reality for pain management in cardiac surgery. Cyberpsychol Behav Soc Netw. 2014;17(6):371-378. doi:10.1089/ cyber.2014.0198
40. McSherry T, Atterbury M, Gartner S, Helmold E, Searles DM, Schulman C. Randomized, crossover study of immersive virtual veality to decrease opioid use during painful wound care procedures in adults. J Burn Care Res. 2018;39(2):278-285. doi:10.1097/ BCR.0000000000000589

41. Sullivan GM, Feinn R. Using effect size-or why the p-value is not enough. J Grad Med Educ. 2012;4(3):279-282. doi:10.4300/JGMED-12-00156.1

42. Harvie DS, Broecker M, Smith RT, Meulders A, Madden VJ, Moseley GL. Bogus visual feedback alters onset of movementevoked pain in people with neck pain. Psychol Sci. 2015;26 (4):385-392. doi:10.1177/0956797614563339

43. Wiederhold BK, Gao K, Sulea C, Wiederhold MD. Virtual reality as a distraction technique in chronic pain patients. Cyberpsychol Behav Soc Netw. 2014;17(6):346-352. doi:10.1089/cyber.2014.0207

44. Jones T, Moore T, Choo J. The impact of virtual reality on chronic pain. PLoS One. 2016;11(12):e0167523. doi:10.1371/journal. pone. 0167523

45. Jin W, Choo A, Gromala D, Shaw C, Squire P. A virtual reality game for chronic pain management: a randomized, controlled clinical study. Stud Health Technol Inform. 2016;220:154-160.

46. Powell W, Simmonds MJ. Virtual reality and musculoskeletal pain: manipulating sensory cues to improve motor performance during walking. Cyberpsychol Behav Soc Netw. 2014;17(6):390-396. doi:10.1089/cyber.2014.0061

47. Sarig Bahat H, Takasaki H, Chen X, Bet-Or Y, Treleaven J. Cervical kinematic training with and without interactive VR training for chronic neck pain - a randomized clinical trial. Man Ther. 2015;20 (1):68-78. doi:10.1016/j.math.2014.06.008

48. Osumi M, Ichinose A, Sumitani M, et al. Restoring movement representation and alleviating phantom limb pain through short-term neurorehabilitation with a virtual reality system. Eur J Pain. 2017;21 (1):140-147. doi:10.1002/ejp.910

49. Roosink M, Robitaille N, Jackson PL, Bouyer LJ, Mercier C. Interactive virtual feedback improves gait motor imagery after spinal cord injury: an exploratory study. Restor Neurol Neurosci. 2016;34 (2):227-235. doi:10.3233/RNN-150563

50. Ichinose A, Sano Y, Osumi M, Sumitani M, Kumagaya S-I, Kuniyoshi Y. Somatosensory feedback to the cheek during virtual visual feedback therapy enhances pain alleviation for phantom arms. Neurorehabil Neural Repair. 2017;31(8):717-725. doi:10.1177/ 1545968317718268

51. Witmer BG, Singer MJ. Measuring presence in virtual environments: a presence questionnaire. Pres Teleoperators Virtual Environ. 1998;7 (3):225-240. doi:10.1162/105474698565686

52. Kern C Dropping virtual reality prices yields better opportunities for healthcare. Health IT Outcomes website. Available from: https:// www.healthitoutcomes.com/doc/dropping-virtual-reality-pricesyields-better-opportunities-for-healthcare-0001. Published 2016. Accessed September 25, 2018.

53. Dascal J, Reid M, IsHak WW, et al. Virtual reality and medical inpatients: a systematic review of randomized, controlled trials. Innov Clin Neurosci. 2017;14(1-2):14-21.

54. Li A, Montaño Z, Chen VJ, Gold JI. Virtual reality and pain management: current trends and future directions. Pain Manag. 2011;1 (2):147-157. doi:10.2217/pmt.10.15 


\section{Publish your work in this journal}

The Journal of Pain Research is an international, peer reviewed, open access, online journal that welcomes laboratory and clinical findings in the fields of pain research and the prevention and management of pain. Original research, reviews, symposium reports, hypothesis formation and commentaries are all considered for publication. The manuscript management system is completely online and includes a very quick and fair peer-review system, which is all easy to use. Visit http:// www.dovepress.com/testimonials.php to read real quotes from published authors.

Submit your manuscript here: https://www.dovepress.com/journal-of-pain-research-journal 